\title{
On Infinite EPR-like Correlations
}

\author{
Leszek Wroński and Tomasz Placek*
}

October 25, 2018

\begin{abstract}
The paper investigates, in the framework of branching space-times, whether an infinite EPR-like correlation which does not involve finite EPR-like correlations is possible.
\end{abstract}

\section{Introduction}

The question addressed in the present paper is the following: Is an infinite EPR-like correlation which does not involve finite EPR-like correlations possible? To explain what finite / infinite refers to in this context, recall the essence of the Einstein-Podolski-Rosen's (1935) set-up: two particles emerging from a source are subjected to position (or momentum) measurements at distant locations in such a way that the two measurement events are spacelike separated. The result is a (perfect) correlation between outcomes of position measurements (or momentum measurements): if the position measured on the first particle is such-and-such, then the position measured on the second particle is certainly such-and-such. The correlation is finite, as it refers to outcomes of two measurements, performed, say, at the left and at the right station. In the subsequent investigations of EPR-like correlations (and of Bell's theorem), set-ups with more measurement stations were considered. Thus, Mermin (1990) considers correlated triples of outcomes of spin measurements - in this case a trio of outgoing particles is subjected

\footnotetext{
${ }^{*}$ We read earlier versions of this paper at the seminar 'Chaos and Quantum Information' held at the Jagiellonian University in Kraków on April 16, 2007 and at the seminar 'On Determinism' held at the University of Bonn on April 20, 2007. For comments and valuable discussions we are grateful to the audiences, and in particular, to Dr. Thomas Müller. Authors' address: Department of Philosophy, Jagiellonian University, Grodzka 52, PL 31-044 Kraków, Poland; LW's email: elwro1@gmail.com and TP's email: uzplacek@cyf-kr.edu.pl.
} 
to spin measurements at three stations. And Greenberger et al. (1989) investigate a set-up with four particles and hence: four measurement stations and quadruples of correlated outcomes. Accordingly, in our usage, the size of a correlation (and hence the distinction: finite vs. infinite) refers to the number of correlated measurement outcomes.

Clearly, the crux of our problem is the notion of possibility, as it occurs in our question. It is perhaps interesting to learn that an infinite EPR-like correlation which does not involve finite EPR-like correlations is logically / mathematically possible; yet, we are after a sharper notion of possibility. On the other extreme, our investigations have nothing to do with experimental possibility, i.e. the possibility of producing an experimental set-up for infinite correlations. Somewhat similarly, we leave aside the question whether quantum theory permits infinite EPR-like correlations. Instead we focus on what we take to be essential aspects of EPR-like correlations: its spatiotemporal and modal features. As for the former, we assume that the experiment occurs in Minkowski space-time. The modal aspect is seen in the popular diagnosis of EPR, which says that although every outcome of a single measurement is possible, certain combinations of outcomes (or, equivalently, a joint outcome) is impossible. To illustrate, although + as well as - are possible outcomes of spin measurement, as performed on one particle, the outcome ++ , that is + on both the particles, is impossible. A final word of warning: we neglect the probabilistic aspect of EPR-like correlations, and we do it for two reasons. First, we focus on perfect correlations and anti-correlations, and we read the extreme probabilities as 'it must happen / it cannot happen'. Second, we believe that in EPR correlations the modal aspect has a conceptual priority over the probabilistic one.

Given the above assumptions, the essence of EPR-like correlations is as follows: in Minkowski space-time, there is a number (finite or not) of measurement events, every two of which are space-like separated. For each measurement event there is a set of possible 'single' outcomes. Yet, certain combinations of single outcomes are impossible.

EPR-like correlations, as described above, can be rigorously investigated in (a non-probabilistic version of) branching space-times (BST), a theory proposed by Belnap (1992) 1 The BST framework rigorously combines modality and (rudiments of) special relativity. It has been used to diagnose Bell's theorem 2 In this theory, a feature analogous to pre-probabilistic EPR-like correlation is called "modal funny business", which is defined so as to capture the idea that a certain combination of otherwise possible outcomes of space-

\footnotetext{
${ }^{1}$ For the updated version of this paper, see its "postprint".

${ }^{2}$ Cf. Belnap and Szabó (1996) and Placek (2000).
} 
like separated measurement events is impossible. The investigation of this notion brought Müller (2005) to ask whether infinite modal funny business which would not involve finite modal funny business was possible. It is this question that sparked our interest in the issues discussed here. In a sense, the question was answered in the positive by Müller, Belnap, and Kohei (2006). They produced a set-theoretical structure (called M2 and described here in Section 4) which satisfies all the axioms of BST and exhibits infinite modal funny business without there being finite modal funny business. Yet, the structure has no relation to any space-time, incl. Minkowski space-time. Thus, the intriguing question remains: namely, can there be in Minkowski space-time a case of infinite modal funny business which does not involve finite modal funny business?

This state of affairs brings in our first task. Contrary to first appearances, BST has models the possible histories (analogous to Lewis-style possible worlds) of which are not associated with Minkowski space-time (or even any space-time). Thus, to investigate whether infinite EPR-like correlations are possible, we had better single out those BST models in which histories are isomorphic to Minkowski space-times. We call such models Minkowskian branching structures (MBS for short). In defining this notion we follow the lead of Müller (2002), yet with two crucial diversions. First, we remove Müller's finiteness assumptions, as they prohibit introduction of 'interesting' infinite structures. Second, we improve on Müller's failed proof of the most desired feature of MBS, namely that every history is isomorphic to Minkowski space-time. To this end we assume a certain topological postulate.

As our second task, we single out two postulates such that each generates infinite funny business in an arbitrary BST model, and such that if none holds in a BST model, the model is free from infinite funny business.

We finally show that in an MBS, if there is infinite funny business, a set in $\Re^{4}$ which generates it must be very strangely located. We also show that truth of the first postulate (A) requires a converging sequence of measurement events. We finally exhibit an MBS model in which the other postulate (B) is true, yet the model has an odd feature, which (we conjecture) is necessary for the truth of the postulate. Thus, our findings strongly say against a possibility of INFFB in physically motivated models of BST.

The paper is organized as follows. In Section 2 we review some definitions and facts of BST which we need later. Section 3 defines and discusses Minkowskian branching structures. Section 4 provides definitions of modal funny business for general BST, introduces two postulates, and relates the occurrence / non-occurrence of infinite funny business in BST to the satisfaction (or not) of those postulates. Section 5 links the above to Minkowskian branching structures: it asks what the postulates presuppose of MBS, and 
hence, for what price one can have in Minkowski space-time an infinite EPRlike correlation which does not involve finite correlations. The final section 6 states our conclusions and poses some open problems.

\section{Branching Space-Times}

The theory of Branching Space-Times (BST), as presented by Nuel Belnap in 1992 Belnap (1992), combines objective indeterminism and relativity in a rigorous way. Its primitives are a nonempty set $W$ (called "Our World", interpreted as the set of all possible point events) and a partial ordering $\leqslant$ on $W$, interpreted as a "causal order" between point events.

There are no "Possible Worlds" in this theory; there is only one world, Our World, containing all that is (timelessly) possible. Instead, a notion of "history" is used, as defined below:

Definition $1 A$ set $h \subseteq W$ is upward-directed iff $\forall e_{1}, e_{2} \in h \exists e \in h$ such that $e_{1} \leqslant e$ and $e_{2} \leqslant e$.

$A$ set $h$ is maximal with respect to the above property iff $\forall g \in W$ such that $g \nsupseteq h, g$ is not upward-directed.

A subset $h$ of $W$ is a history iff it is a maximal upward-directed set.

For histories $h_{1}$ and $h_{2}$, any maximal element in $h_{1} \cap h_{2}$ is called a choice point for $h_{1}$ and $h_{2}$.

A very important feature of BST is that histories are closed downward: if $e_{1} \leqslant e_{2}$ and $e_{1} \notin h$, then $e_{2} \notin h$. In other words, there is no backward branching among histories in BST. No two incompatible events are in the past of any event; equivalently: the past of any event is "fixed", containing only compatible events.

We will now give the definition of a BST model; for more information about BST in general see Belnap (1992).

Definition $2\langle W, \leqslant\rangle$ where $W$ is a nonempty set and $\leqslant i$ is a partial ordering on $W$ is a model of BST if and only if it meets the following requirements:

1. The ordering $\leqslant$ is dense.

2. S has no maximal elements.

3. Every lower bounded chain in $W$ has an infimum in $W$.

4. Every upper bounded chain in $W$ has a supremum in every history that contains it. 
5. (Prior choice principle ('PCP')) For any lower bounded chain $O \in$ $h_{1}-h_{2}$ there exists a point $e \in W$ such that $e$ is maximal in $h_{1} \cap h_{2}$ and $\forall e^{\prime} \in O e<e^{\prime}$.

\section{Introducing Minkowskian Branching Struc- tures}

BST is a frugal theory, since it allows for models whose histories hardly have spacetimes, and the only 'spatiotemporal' notion is that of the ordering $\leqslant$. We are going to single out a class of BST models, in which histories occur in spacetimes, and moreover, all these spacetimes are Minkowskian. This part of our work is based on Müller's [2002] theory.

The points of the Minkowskian space-time are elements of $\mathbb{R}^{4}$, e.g. $x=$ $\left\langle x^{0}, x^{1}, x^{2}, x^{3}\right\rangle$, where the first element of the quadruple is the time coordinate. The Minkowskian space-time distance (Lorentz interval) is a function $D_{M}^{2}: \mathbb{R}^{4} \times \mathbb{R}^{4} \rightarrow \mathbb{R}$ defined as follows (for $x, y \in \mathbb{R}^{4}$ ):

$$
D_{M}^{2}(x, y):=-\left(x^{0}-y^{0}\right)^{2}+\sum_{i=1}^{3}\left(x^{i}-y^{i}\right)^{2}
$$

The natural ordering on the Minkowski space-time, call it "Minkowskian ordering $\leqslant{ }_{M}$ ", is defined as follows $\left(x, y \in \mathbb{R}^{4}\right)$ :

$$
x \leqslant_{M} y \text { iff } D_{M}^{2}(x, y) \leqslant 0 \text { and } x^{0} \leqslant y^{0}
$$

We will say that two points $x, y \in \mathbb{R}^{4}$ are space-like related ("SLR" for short) iff neither $x \leqslant_{M} y$ nor $y \leqslant_{M} x$. Naturally, $x<_{M} y$ iff $x \neq y$ and $x \leqslant_{M} y$.

Now we need to provide a framework for "different ways in which things can happen" and for filling the space-times with content. For the first task we will need a set $\Sigma$ of labels $\sigma, \eta, \ldots$. (In contrast to Müller (2002), we allow for any cardinality of $\Sigma$ ). For the second task, we will use a so called "state" function $S: \Sigma \times \mathbb{R}^{4} \rightarrow P$, where $P$ is a set of point properties (on this we just quote Müller saying "finding out what the right $P$ is is a question of physics, not one of conceptual analysis").

One could ask about the reasons for an extra notion of a "scenario". Why don't we build histories just out of points from $\mathbb{R}^{4} \times P$ ? The reason is that a member of BST's Our World has a fixed past. If two different trains of events lead to exactly the same event $E \in \mathbb{R}^{4} \times P$, the situation gives rise to two different point events, two different members of $W$. In contrast: for 
a point $\left\langle x, p_{0}\right\rangle$ from $\mathbb{R}^{4} \times P$ there can exist two different points $\left\langle y, p_{1}\right\rangle$ and $\left\langle y, p_{2}\right\rangle$ from $\mathbb{R}^{4} \times P$ such that $y<_{M} x$. This would be a case of backward branching, so the set $\mathbb{R}^{4} \times P$ is not a good candidate for the master set $W$ of any BST model.

The idea behind the concept of scenario is that every scenario corresponds to a $\mathbb{R}^{4}$ space filled with content 3 , where the content derives from the elements of $P$. Assuming a certain state function $S$ is given, for any $\sigma, \eta \in \Sigma$ the set $C_{\sigma \eta} \subset \mathbb{R}^{4}$ is the set of "splitting points" between scenarios $\sigma$ and $\eta$, intuitively: the set of points in which a choice between the two scenarios is made. All members of $C_{\sigma \eta}$ have to be space-like related. Of course a choice between $\sigma$ and $\eta$ is a choice between $\eta$ and $\sigma$, so $C_{\sigma \eta}=C_{\eta \sigma}$. The BST axiom of prior choice principle motivates our postulate that any two different scenarios split. Formally: $\forall \sigma, \eta \in \Sigma\left(\sigma \neq \eta \Rightarrow C_{\sigma \eta} \neq \emptyset\right)$.

The next requirement concerns triples of scenarios. Any set $C_{\sigma \eta}$ determines a region in which both scenarios coincide: namely, that part of $\mathbb{R}^{4}$ that is not in the Minkowskian sense strictly above any point from $C_{\sigma \eta}$. Following Müller we call it the region of overlap $R_{\sigma \eta}$ between scenarios $\sigma, \eta$ defined as below:

$$
R_{\sigma \eta}:=\left\{x \in \mathbb{R}^{4} \mid \neg \exists y \in C_{\sigma \eta} y<_{M} x\right\}
$$

(Of course it follows that for any $\sigma, \eta \in \Sigma C_{\sigma \eta} \subseteq R_{\sigma \eta}$.) Assuming the sets $C_{\sigma \eta}$ and $C_{\eta \gamma}$ are given, we get two regions of overlaps $R_{\sigma \eta}$ and $R_{\eta \gamma}$. At the points in the intersection of those two regions $\sigma$ coincides with $\eta$ and $\eta$ coincides with $\gamma$, therefore by transitivity $\sigma$ coincides with $\gamma$. In general we can say that for any $\sigma, \eta, \gamma \in \mathbb{R}^{4}$

$$
R_{\sigma \gamma} \supseteq R_{\sigma \eta} \cap R_{\eta \gamma}
$$

which translated to a requirement on sets of splitting points is

$$
\forall x \in C_{\sigma \gamma} \exists y \in C_{\sigma \eta} \cup C_{\eta \gamma} y \leqslant_{M} x .
$$

In his paper Müller put another requirement on $C_{\sigma \eta}$ : finitude. The motivation was to exclude splitting along a "simultaneity slice". The strong requirement of finitude excludes however many more types of situations, in which splitting is not continuous or happens in a region of space-time of a finite diameter. In the present paper we drop this requirement, not putting any restrictions on the cardinality of $C_{\sigma \eta}$ for any $\sigma, \eta \in \Sigma$. As a sidenote, this leads to the fact that in some models there may be choice points which are not intuitively connected with any splitting point. For details, see 7.1 in the Appendix.

\footnotetext{
${ }^{3}$ Fix a scenario $\alpha$. The above mentioned corresponding space filled with content is $A \subseteq \mathbb{R}^{4} \times P$ such that $\langle x, p\rangle \in A$ iff $S(\alpha, x)=p$.
} 
The state function assigns to each pair $\left\langle\right.$ a label from $\Sigma$, a point from $\left.\mathbb{R}^{4}\right\rangle$ an element of $P$. Colloquially, the state function tells us what happens at a certain point of the space-time in a given scenario.

After Müller (2002), we now proceed to construct the elements of MBS version of Our World; they will be equivalence classes of a certain relation $\leqslant_{S}$ on $\Sigma \times \mathbb{R}^{4}$. For convenience, we write the elements of $\Sigma \times \mathbb{R}^{4}$ as $x_{\sigma}$ where $x \in \mathbb{R}^{4}, \sigma \in \Sigma$. The idea is to "glue together" points in regions of overlap; hence the relation is defined as below:

$$
x_{\sigma} \equiv_{S} y_{\eta} \text { iff } x=y \text { and } x \in R_{\sigma \eta}
$$

Müller provides a simple proof of the fact that $\equiv_{S}$ is an equivalence relation on $\Sigma \times \mathbb{R}^{4}$; therefore we can produce a quotient structure. The result is the set $B$ being the MBS version of Our World:

$$
B:=\left(\Sigma \times \mathbb{R}^{4}\right) / \equiv_{S}=\left\{\left[x_{\sigma}\right] \mid \sigma \in \Sigma, x \in \mathbb{R}^{4}\right\} .
$$

where $\left[x_{\sigma}\right]$ is the equivalence class of $x$ with respect to the relation $\equiv_{S}$ :

$$
\left[x_{\sigma}\right]=\left\{x_{\eta} \mid x_{\sigma} \equiv_{S} x_{\eta}\right\} \text {. }
$$

Next, we define a relation $\leqslant_{S}$ on $B$ :

$$
\left[x_{\sigma}\right] \leqslant_{S}\left[y_{\eta}\right] \text { iff } x \leqslant_{M} y \text { and } x_{\sigma} \equiv_{S} x_{\eta}
$$

which (as Müller shows) is a partial ordering on $B$.

The goal would now be to prove that $\left\langle B, \leqslant_{S}\right\rangle$ is a model of BST. To do so, and in particular to prove the prior choice principle and requirement no. 4 from definition 2, we need to know more about the shape of the histories in MBS - that they are the intended ones.

\subsection{The shape of MBS histories}

We would like histories, that is: maximal upward-directed sets, to be sets of equivalence classes $\left[x_{\sigma}\right]$ (with respect to $\equiv_{S}$ ) for $x \in \mathbb{R}^{4}$ for some $\sigma \in \Sigma$. In other words, we wish to unambiguously refer to any history by a label from $\Sigma$, requiring one-to-one correspondence of the sets of histories and labels. This is Müller's [2002] Lemma 3 and our

Theorem 3 Every history in a given $M B S$ is of the form $h=\left\{\left[x_{\sigma}\right] \mid x \in \mathbb{R}^{4}\right\}$ for some $\sigma \in \Sigma$.

\footnotetext{
${ }^{4}$ We can look at the situation from a slightly different perspective: every label $\sigma$ is assigned a mapping $S_{\sigma}$ from $\mathbb{R}^{4}$ to $P$; see also previous footnote.
} 
The problem is that, aside from minor brushing up required by the proof of the "right" direction, the proof of the "left" direction supplied in Müller (2002) needs to be fixed as it does not provide adequate reasons for nonemptiness of an essential intersection $\bigcap \Sigma_{h}\left(z_{i}\right)$. More on that below. Let us divide the above theorem into two lemmas (4) and 8) corresponding to the directions and prove the "right" direction first. Until we prove the theorem we refrain from using the term "history" and substitute it with a "maximal upward-directed set" for clarity.

Lemma 4 If $h=\left\{\left[x_{\sigma}\right] \mid x \in \mathbb{R}^{4}\right\}$ for some $\sigma \in \Sigma$ than $h$ is a maximal upward-directed subset of $B$.

Proof: Let us consider $e_{1}, e_{2} \in h, e_{1}=\left[x_{\sigma}\right], e_{2}=\left[y_{\sigma}\right]$. Since $x, y \in \mathbb{R}^{4}$ there exists a $z \in \mathbb{R}^{4}$ such that $x \leqslant_{M} z$ and $y \leqslant_{M} z$. Therefore $\left[x_{\sigma}\right] \leqslant_{S}\left[z_{\sigma}\right]$ and $\left[y_{\sigma}\right] \leqslant_{S}\left[z_{\sigma}\right]$, and so $h$ is upward-directed.

For maximality, consider a $g \subseteq B, g \nsupseteq h$ and assume $g$ is upward-directed. It follows that there exists a point $\left[x_{\eta}\right] \in g-h$ such that $\left[x_{\eta}\right] \neq\left[x_{\sigma}\right] \in h$. Since both points belong to $g$ which is upward-directed, there exists $\left[z_{\alpha}\right] \in g$ (note that we are not allowed to choose $\sigma$ as the index at that point) such that $\left[x_{\eta}\right] \leqslant_{S}\left[z_{\alpha}\right]$ and $\left[x_{\sigma}\right] \leqslant_{S}\left[z_{\alpha}\right]$. Therefore $x_{\eta} \equiv_{S} x_{\alpha} \equiv_{S} x_{\sigma}$, and so we arrive at a contradiction by concluding that $\left[x_{\eta}\right]=\left[x_{\sigma}\right]$. Q.E.D.

The proof of the other direction is more complex and, what might be surprising, involves a topological postulate. First, we will need a simple definition:

Definition 5 For a given maximal upward-directed set $h$ and a point $x \in \mathbb{R}^{4}$, $\Sigma_{h}(x):=\left\{\sigma \in \Sigma \mid\left[x_{\sigma}\right] \in h\right\}$.

Consider now a given maximal upward-directed set $h \subseteq B$. With every lower bounded chain $L \subset \mathbb{R}^{4}$ we would like to associate a topology (called "chain topology") on the set of $\Sigma_{h}(\inf (L))$. We define the topology by describing the whole family of closed sets, which is equal to $\left\{\emptyset, \Sigma_{h}(\inf (L))\right\} \cup$ $\left\{\Sigma_{h}(l) \mid l \in L\right\} \cup\left\{\cap\left\{\Sigma_{h}(l) \mid l \in L\right\}\right\}$. (Because $L$ is a chain it is evident that the family is closed with respect to intersection and finite union). The postulate runs as follows:

Postulate 6 For every maximal upward-directed set $h \subseteq B$ and for every lower bounded chain $L \subset \mathbb{R}^{4}$ the "chain topology" described above is compact.

It is easily verifiable that in such a topology $\left\{\Sigma_{h}(l) \mid l \in L\right\}$ is a centred family of closed sets (every finite subset of it has a nonempty intersection). Together with the above postulate we get this result: 
Corollary 7 For every maximal upward-directed set $h \subseteq B$ and for every chain $L \subset \mathbb{R}^{4}, \bigcap\left\{\Sigma_{h}(l) \mid l \in L\right\} \neq \emptyset$.

Lemma 8 If $h$ is a maximal upward-directed subset of $B$ then $h=\left\{\left[x_{\sigma}\right] \mid x \in\right.$ $\left.\mathbb{R}^{4}\right\}$ for some $\sigma \in \Sigma$.

The structure of the proof mimics the proof of Müller's (see Müller (2002)). It is divided into three parts, the first and the last being reproduced here. On the other hand, the second part contains an error (as stated above, the statement that $\bigcap \Sigma_{h}\left(z_{i}\right) \neq \emptyset$ is not properly justified) and bears on an assumption that for every history $h$ and point $x \in \mathbb{R}^{4}$ the set $\Sigma_{h}(x)$ is at most countably infinite. We wish both to drop this assumption and correct the proof using the above topological postulate.

Proof: Suppose that $h$ is a maximal upward-directed subset of $B$. In order to prove the lemma, we will prove the following three steps:

1. If for some $\sigma, \eta \in \Sigma$ both $\left[x_{\sigma}\right] \in h$ and $\left[x_{\eta}\right] \in h$, then $x_{\sigma} \equiv_{S} x_{\eta}$.

2. There is a $\sigma \in \Sigma$ such that for every $\eta$, if $\left[x_{\eta}\right] \in h$, then $x_{\eta} \equiv_{S} x_{\sigma}$.

3. With the $\sigma$ from step $2, h=\left\{\left[x_{\sigma}\right] \mid x \in \mathbb{R}^{4}\right\}$.

Ad. 1. Since $h$ is maximal by assumption, there exists a $\left[y_{\gamma}\right] \in h$ such that $\left[x_{\sigma}\right] \leqslant_{S}\left[y_{\gamma}\right]$ and $\left[x_{\eta}\right] \leqslant_{S}\left[y_{\gamma}\right]$. These last two facts imply that $x_{\sigma} \equiv_{S} x_{\gamma} \equiv_{S} x_{\eta}$, so by transitivity of $\equiv_{S}$ we get $x_{\sigma} \equiv_{S} x_{\eta}$.

Ad. 2. Assume the contrary: $\forall \sigma \in \Sigma \exists\left[x_{\eta}\right] \in h, x_{\eta} \not_{S} x_{\sigma}$.

Take a point $\left[y_{\kappa}\right] \in h$. Accordingly, $\Sigma_{h}(y) \neq \emptyset$.

For each scenario $\sigma_{\alpha} \in \Sigma_{h}(y)$ we define a set $\Theta_{\alpha}=\left\{x \in \mathbb{R}^{4} \mid \exists \eta \in\right.$ $\left.\Sigma_{h}(y):\left[x_{\eta}\right] \in h \wedge x_{\sigma_{\alpha}} \not \equiv_{S} x_{\eta}\right\}$, which by our assumption is never empty. Colloquially, it is a set of the points that make the scenario a wrong candidate for the proper scenario from our lemma - the scenario "doesn't fit" the history at those points. For each scenario $\sigma_{\alpha}$ we would like to choose a single element of $\Theta_{\alpha}$, and to that end we employ a choice function $T$ defined on the set of subsets of $\mathbb{R}^{4}$ such that $T\left(\Theta_{\alpha}\right) \in \Theta_{\alpha}$, naming the element chosen by it as follows: $T\left(\Theta_{\alpha}\right):=x_{\alpha} \cdot 5$

Observe that we will arrive at a contradiction if we prove that

$$
\bigcap_{\sigma_{\alpha} \in \Sigma_{h}(y)} \Sigma_{h}\left(x_{\alpha}\right) \neq \emptyset
$$

(since for any $\sigma_{\beta} \in \Sigma_{h}(y) \sigma_{\beta} \notin \Sigma_{h}\left(x_{\beta}\right)$ ). In order to apply our topological postulate, we will construct a chain $L=\left\{z_{0}, z_{1}, \ldots, z_{\omega}, \ldots\right\}$ of points in $\mathbb{R}^{4}$.

\footnotetext{
${ }^{5}$ Bear in mind that since $\alpha$ is a number serving just as an index for scenarios, $x_{\alpha}$ (like $x_{\beta}$ in the line below inequality 10] is a point from $\mathbb{R}^{4}$ and does not denote a point - scenario pair.
} 
It will be lower bounded by its initial element $z_{0}$. Moreover, we want it to be vertical, since this way it will (if it does not have an upper bound itself) contain an upper bound of any point in $\mathbb{R}^{4}$, which will be needed in our proof.

We first define a function " $u p$ " which given two points $a, b \in \mathbb{R}^{4}$ will produce a point $c \in \mathbb{R}^{4}$ such that $c$ has the same spatial coordinates as $a$ but is above $b$. In other words, if $a=\left\langle a^{0}, a^{1}, a^{2}, a^{3}\right\rangle \in \mathbb{R}^{4}, b=\left\langle b^{0}, b^{1}, b^{2}, b^{3}\right\rangle \in \mathbb{R}^{4}$, $u p(a, b):=\left\langle a^{0}+\left(\sum_{1}^{3}\left(a^{i}-b^{i}\right)^{2}\right)^{1 / 2}, a^{1}, a^{2}, a^{3}\right\rangle \in \mathbb{R}^{4}$. Notice that up is not commutative.

We proceed to define the above mentioned chain $L$ in the following way:

1. $z_{0}=u p\left(y, x_{0}\right)$.

$z_{1}=u p\left(z_{0}, x_{1}\right)$.

Generally, $z_{k+1}=u p\left(z_{k}, x_{k+1}\right)$.

2. Suppose $\rho$ is a limit number. Define $A_{\rho}:=\left\{z_{\beta} \mid \beta<\rho\right\} 6$. As you can see, $A_{\rho}$ is the part of our chain we have managed to construct so far. We need to distinguish two cases:

a) $A_{\rho}$ is upper bounded with respect to $\leqslant_{M}$. Then it has to have "vertical" upper bounds $t_{0}, t_{1} \ldots$ with spatial coordinates $t_{n}^{i}=z_{0}^{i}(i=1,2,3)$. In this case, we employ the above defined function $T$ to choose one of the upper bounds of $A_{\rho}$ :

$$
t_{\rho}:=T\left(\left\{t \in \mathbb{R}^{4} \mid \forall \beta<\rho z_{\beta} \leqslant_{M} t \wedge t^{i}=z_{0}^{i}(i=1,2,3)\right\}\right) .
$$

Then we put $z_{\rho}:=u p\left(t_{\rho}, x_{\rho}\right)$, arriving at the next element of our chain $L$.

b) if $A_{\rho}$ is not upper bounded with respect to $\leqslant_{M}$, then no matter which point in $\mathbb{R}^{4}$ we choose, it is possible to find a point from $A_{\rho}$ above it (since $A_{\rho}$ is vertical). Therefore the set

$$
B_{\rho}=\left\{t \in A_{\rho} \mid x_{\rho} \leqslant{ }_{M} t\right\}
$$

is not empty. We put $\left[z_{\rho}\right]:=T\left(B_{\rho}\right)$, arriving at the next element of our chain $L$.

Notice that in our chain it might happen that while $\alpha<\beta, z_{\beta} \leqslant{ }_{M} z_{\alpha}$, but $z_{0}$ is a lower bound of $L$. Therefore our postulate 6 applies. By employing it and corollary 7 we infer that

$$
\bigcap_{\sigma_{\alpha} \in \Sigma_{h}(y)}\left\{\Sigma_{h}\left(z_{\alpha}\right) \mid z_{\alpha} \in L\right\} \neq \emptyset
$$

By our construction of the chain $L$, for all $\alpha$ it is true that $x_{\alpha} \leqslant_{M} z_{\alpha}$. Therefore $\Sigma_{h}\left(z_{\alpha}\right) \subseteq \Sigma_{h}\left(x_{\alpha}\right)$. Thus, from 13 we immediately get

$$
\bigcap_{\sigma_{\alpha} \in \Sigma_{h}(y)} \Sigma_{h}\left(x_{\alpha}\right) \neq \emptyset
$$

\footnotetext{
${ }^{6}$ Again: $\beta$ is just an index, not a scenario, so $A_{\rho}$ is a subset of $\mathbb{R}^{4}$.
} 
which is the equation 10 that we tried to show. Therefore we arrive at a contradiction and part 2 of the proof is complete.

Ad. 3. We have shown that there is a scenario $\sigma \in \Sigma$ such that all members of $h$ can be identified as $\left[x_{\sigma}\right]$ for some $x \in \mathbb{R}^{4}$. What remains is to show that the history cannot "exclude" some regions of $\{\sigma\} \times \mathbb{R}^{4}$, that is: to prove that for all $x \in \mathbb{R}^{4},\left[x_{\sigma}\right] \in h$. But in lemma 4 we have shown that $\left\{\left[x_{\sigma}\right] \mid x \in \mathbb{R}^{4}\right\}$ is a maximal upward-directed subset of $B$, so any proper subset of it cannot be maximal upward-directed. Q.E.D.

By showing lemmas 4 and 8 we have proved theorem 3 .

\subsection{The importance of the topological postulate}

So far it might seem that our topological postulate 6 is just a handy trick for proving the lemma 8 . To show its importance we will now prove that its falsity leads to the falsity of the lemma, and then present an example of a structure in which the lemma does not hold.

Theorem 9 If the postulate 6 is false, then lemma 8 is also false.

Proof: Assume that our topological postulate does not hold. Therefore there exists a maximal upward-directed set $h \subseteq B$ and a lower bounded chain $L \subset \mathbb{R}^{4}$ such that the chain topology is not compact. This is by rules of topology equivalent to the fact that there is a centred family of closed sets with an empty intersection. But all closed sets in the chain topology form a chain with respect to inclusion. Of course, if a part of a chain has an empty intersection, a superset of the part also has an empty intersection. We infer that

$$
\bigcap_{x \in L} \Sigma_{h}(x)=\emptyset
$$

from which, by definition 5, we get that

$$
\neg \exists \sigma \in \Sigma: \forall x \in L\left[x_{\sigma}\right] \in h
$$

so there is no scenario $\sigma$ such that $h=\left\{\left[x_{\sigma}\right] \mid x \in \mathbb{R}^{4}\right\}$. Thus, lemma 8 is false. Q.E.D.

In the Appendix (section 7.2) we show a situation in which lemma 8 does not hold. The construction resembles the $M_{1}$ structure from Müller et al. (2006). 


\subsection{BST models and MBS}

Having proven theorem 3 we can adopt Müller's proof (from Müller (2002)) of the fact that $\left\langle B, \leqslant_{S}\right\rangle$ meets all the requirements in definition 2 and conclude that it is a model of BST. We keep in mind, though, that we have introduced a new postulate 6 into the proof and shown that it is not trivial (not always true). We will demand from the structures we would like to call "Minkowskian Branching Structures" to meet our topological postulate. This way, a MBS is a special kind of a BST model: its Our World and ordering $\leqslant$ are constructed as respectively $B$ and $\leqslant_{S}$ as proposed by Müller, and furthermore our postulate 6 is true in the model.

Due to the following self-evident Fact, we have fulfilled our promise from the introduction and produced BST models in which histories are isomorphic to Minkowski space-times.

Fact 10 Let $\mathcal{W}=\left\langle W, \leqslant_{S}\right\rangle$ be an $M B S$ and let $h$ be a history in $\mathrm{W}$ of the form $\left\{\left[x_{\sigma}\right] \mid x \in \mathbb{R}^{4}\right\}$ for a certain $\sigma \in \Sigma$. Then

$$
\left\langle h, \leqslant\left._{S}\right|_{h}\right\rangle \cong\left\langle\mathbb{R}^{4}, \leqslant_{M}\right\rangle
$$

by means of the isomorphism $i: h \rightarrow \mathbb{R}^{4}$ such that $i\left(\left[x_{\sigma}\right]\right)=x$.

\section{Funny business}

The rest of the paper concerns the funny business phenomenon in its finitary and infinitary variants. Funny business in BST is to resemble EPR correlations. The underlying idea is that there are two space-like related events whose outcomes are correlated in the sense that a combinatorially possible history is missing. As an example, consider a BST model of the EPR-Bohm experiment (Bohm, 1951). There are two space-like separated measurement events $e_{1}$ and $e_{2}$, idealized to be point-like. Each has two outcomes, 'spin up' and 'spin down', to be written as, resp. $H_{1}+, H_{1}-$ and $H_{2}+, H_{2}-$. Since histories with results 'spin up' and 'spin down' are possible, we assume that the intersections $H_{1}+\cap H_{2}-$ and $H_{1}-\cap H_{2}+$ are nonempty. Yet, we put: $H_{1}+\cap H_{2}+=H_{1}-\cap H_{2}-=\emptyset$, since no history with same spin projections is possible.

Taking a clue from this example, funny business seems to require two SLR point events $e_{1}, e_{2}$ such that for some outcomes $H_{1}$ of $e_{1}$ and $H_{2}$ of $e_{2}$ : $H_{1} \cap H_{2}=\emptyset$. Since $e_{1}$ SLR $e_{2}$, the two share a history. Now, this basic idea could be generalized in two directions, giving rise to the notions of finitary funny business and infinitary funny business. As for the former, following 
Belnap (2002) we allow for extended (i.e., not point-like) events, say $A$ and $B$, require that they are SLR in the sense that $\forall x \in A \forall y \in B(x S L R y)$, and we do not postulate that the sum of the two events to be a subset of a history 7 The result is Belnap's notion of generalized-primary space-likerelated modal-correlation-funny business, i.e., g-p s-l-r m-c funny business for short.

To obtain the infinitary version of funny business, consider a set $S$ of (not necessarily SLR) infinitely many point events and require that any two elements of $S$ be consistent in the sense that every outcome of the first event intersects non-emptily with every outcome of the other; that feature obtains for any finite subset of $S$. The funny business consists in there being a set of outcomes of events of $S$, one outcome for each element of $S$, the intersection of which is empty. The resulting notion, which is closely related to combinatorial funny business of Müller et al. (2006) gives rise to a more familiar concept if one further requires that $S$ is pairwise SLR and is a subset of some history $h$.

To properly define funny business, we will need a few formal notions.

Definition 11 Hist is the set of all histories in the model. $H_{(e)}$ is the set of all histories to which point event e belongs.

For $e_{1}, e_{2} \in W, e_{1} S L R e_{2}$ iff $\exists h \in H i s t: e_{1}, e_{2} \in h$ and $e_{1} \nless e_{2}$ and $e_{2} \nless e_{1}$. For $E_{1}, E_{2} \subseteq W, E_{1} S L R E_{2}$ iff $\forall e_{1} \in E_{1} \forall e_{2} \in E_{2}: e_{1} S L R e_{2}$.

Next, replacing the informal notion of an event's outcome, we have a concept of "elementary possibility at $e$ ", defined as an element of a certain partition of $H_{(e)}$. The partition is an equivalence relation $\equiv_{e}$ on $H_{(e)}$ which is to convey the sense of "being undivided in $e$ " - sharing a point above $e$.

Definition 12 Consider $h_{1}, h_{2} \in H_{(e)} . h_{1} \equiv_{e} h_{2}$ iff $\exists e^{*}>$ e such that $e^{*} \in h_{1} \cap h_{2} . h_{1} \perp_{e} h_{2}$ iff $h_{1}, h_{2} \in H_{(e)}$ and it is not the case that $h_{1} \equiv_{e} h_{2}$.

The relation $\equiv_{e}$ is an equivalence relation on $H_{(e)}$ due to BST postulates, as shown in Belnap (1992), Facts 45-46.

Definition 13 If $h \in H_{(e)}$, we say that $\Pi_{e}\langle h\rangle \subseteq H_{(e)}$ is an elementary possibility (open) in e iff it is the equivalence class of the history h w.r.t. the relation $\equiv_{e}$. If $x \in W$ and $e<x$, by $\Pi_{e}\langle x\rangle$ we mean the elementary possibility in e to which history $h \in H_{(x)}$ belongs.

Following the existing literature we define $\Pi_{e}$ as the set of all elementary possibilities at $e$.

\footnotetext{
${ }^{7}$ The reason for this selection is, in the last instance, the existence of 'nice' theorems following from this concept. For more, cf. footnote ? of Belnap (2002).
} 
Next, for a given set $S$ we will consider functions $f$ which, given a point $e \in S$ as an argument, produce an elementary possibility from $\Pi_{e}$. Colloquially speaking, function $f$ resembles a pointer, indicating for every $e \in S$ which elementary possibility at $e$ is selected. Formally, a pointer function is an element of the set $\prod_{e \in S} \Pi_{e}$ of product functions, defined as follows: :

$$
\prod_{e \in S} \Pi_{e}=\left\{f: S \rightarrow \bigcup_{e \in S} \Pi_{e}: \forall e^{\prime} \in S f\left(e^{\prime}\right) \in \Pi_{e^{\prime}}\right\}
$$

The definitions of no (in)finitary funny business and (in)finitary funny business run as follows:

Definition 14 Assume $S \subseteq W$ and a function $f \in \prod_{e \in S} \prod_{e}$.

$\langle S, f\rangle$ is not a case of finitary funny business iff for any $A_{1}, A_{2} \subseteq S$ : if $A_{1} S L R A_{2}$ and $\bigcap\left\{f(e): e \in A_{i}\right\} \neq \emptyset$ for $i=1,2$, then $\bigcap\{f(e): e \in$ $\left.A_{1} \cup A_{2}\right\} \neq \emptyset$.

$\langle S, f\rangle$ is not a case of infinitary funny business iff $(1) \operatorname{card}(S)<\omega$ or (2) $\exists A(A \subseteq$ fin $S \wedge \forall h \in H i s t ; A \nsubseteq h)$ or (3) if $\forall e, e^{\prime} \in S\left(e<e^{\prime} \rightarrow\right.$ $\left.f\left(e^{\prime}\right) \subseteq f(e)\right)$, then $\bigcap\{f(e): e \in S\} \neq \emptyset$.

$S$ does not give rise to (in)finitary funny business iff $\forall f \in \prod_{e \in S} \prod_{e}$

$\langle S, f\rangle$ is not a case of (in)finitary funny business.

On this definition, $\langle S, f\rangle$ is a case of finitary funny business iff there are $A_{1}, A_{2} \subseteq S$ such that $A_{1} \operatorname{SLR} A_{2}$ and $\bigcap\left\{f(e): e \in A_{i}\right\} \neq \emptyset$ for $i=1,2$ but $\bigcap\left\{f(e): e \in A_{1} \cup A_{2}\right\}=\emptyset$. I.e., this is a case of Belnap's g-p s-l-r m-c funny business (for details, see Appendix).

And $\langle S, f\rangle$ is a case of infinitary funny business iff $(1) \operatorname{card}(S) \geqslant \omega$ and (2) $\forall A(A \subseteq$ fin $S \rightarrow \exists h \in H i s t: A \subseteq h)$, and (3) $\forall e, e^{\prime} \in S\left(e<e^{\prime} \rightarrow\right.$ $\left.f\left(e^{\prime}\right) \subseteq f(e)\right)$ and $(4) \bigcap\{f(e): e \in S\}=\emptyset$. To see the rationale underlying clause (3), note that due to that clause, there is no infinitary funny business if for some $e, e^{\prime} \in S\left(e<e^{\prime} \wedge f\left(e^{\prime}\right) \nsubseteq f(e)\right)$, which entails, by properties of elementary possibilities, that $\left(^{*}\right) f\left(e^{\prime}\right) \cap f(e)=\emptyset$. In other words, the reason why the infinite intersection (4) is empty is that the intersection $\left(^{*}\right)$ is empty. In a similar vein, by clause (2), we do not call it infinitary funny business if some finite subset of $S$ is inconsistent: in this case there is nothing funny in $\bigcap\{f(e): e \in S\}=\emptyset$, as this follows from the above.

The relation to Müller et al. (2006)'s notion of combinatorial funny business is this:

If $\langle S, f\rangle$ is a case of infinitary funny business, then $\langle S, f\rangle$ constitutes a case of combinatorial funny business. In the other direction, if $\langle S, f\rangle$ constitutes a case of combinatorial funny business but $\langle S, f\rangle$ is not a case of finitary funny 
business, then $\langle S, f\rangle$ is a case of infinitary funny business. For details, see Appendix.

One might find the above definitions not completely intuitive or even objectionable, and to some extend we share this feeling. For instance, in what follows, while discussing finitary and infinitary funny business, we are concerned with only such $S$ that are pairwise SLR and a subset of a history. So, the definitions given above are too general for our purposes. Despite these disadvantages, we assume them since they are closely related to the extant definitions, and there are some odd structures arising from $S$ that is neither pairwise SLR nor a subset of a history. For brevity, from now on instead of "finitary funny business" we will usually write "FINFB" and instead of "infinitary funny business" we will usually write "INFFB". We will also say that NO FINFB (NO INFFB) is true in a BST model $\mathcal{W}=\langle W, \leqslant\rangle$ meaning that no $S \subseteq W$ gives rise to FINFB (INFFB).

\section{1 $\quad M_{2}$}

Müller et al. (2006) introduced a certain BST structure named $M_{2}$, in which FINFB was absent, whereas INFFB was present. We will now briefly reproduce their definition, because it is an interesting example of funny business and we will use it in our theorems. For a detailed discussion and a proof that $M_{2}$ is a BST model with the above properties, see the mentioned paper.

$M_{2}$ is a pair $\langle W, \leqslant\rangle$. $W$ is a union of four sets: $W_{0}=(-\infty, 0], W_{1}=$ $(0,1] \times \mathbb{N}, W_{2}=(1,2) \times \mathbb{N} \times\{0,1\}$ and $W_{3}=[2, \infty) \times \mathbb{F}$ where $\mathbb{F}$ is the set of all functions $g: \mathbb{N} \rightarrow\{0,1\}$ such that for only finitely many $n \in \mathbb{N}, g(n)=0$.

The strict partial ordering $<$ is the transitive closure of the following four relations:

- For $e, e_{1}$ from the same $W_{i}: e<e_{1}$ iff the first coordinate of $e$ is smaller than that of $e_{1}$ and the other coordinates are the same.

- $x<(y, n)$ for every $x \in W_{0}$ and $(y, n) \in W_{1}$.

- For $(x, n) \in W_{1}$ and $(y, m, i) \in W_{2}:(x, n)<(y, m, i)$ iff $n=m$.

- For $(x, n, i) \in W_{2}$ and $(y, g) \in W_{3}:(x, n, i)<(y, g)$ iff $(g(n)=i$.

The non-strict companion $\leqslant$ of $<$ is defined as usual: $e \leqslant e^{\prime}$ iff $e<e^{\prime}$ or $e=e^{\prime}$. The structure $M_{2}$ has a countable set Hist of histories and also a countable set of binary choice points $S=\{\langle 1, n\rangle: n \in \mathbb{N}\}$. Moreover, there is one-to-one correspondence between Hist and $\mathbb{F}$, which allows us to identify values of product functions (i.e., elementary possibilities) with certain subsets 
of $\mathbb{F}$. At each point $e=\langle 1, n\rangle$ there are two elementary possibilities, both of the form $\{g \in \mathbb{F} \mid g(n)=b\}$, where $b$ is 0 or 1 . Taking now a product function $f \in \prod_{e \in S} \prod_{e}$ such that $f(\langle 1, n\rangle)=\{g \in \mathbb{F} \mid g(n)=0\}$, it is easy to see that $\langle S, f\rangle$ is a case of INFFB since in $\mathbb{F}$ there is no function $z$ such that $z(x)=0$ for every $x \in \mathbb{N}$. On the other hand, there is no case of FINFB in $M_{2}$ (see Müller et al. (2006)). Thus, $M_{2}$ is just a case in point: it has INFFB that does not involve any case of FINFB. In a due time, we will ask if the structure can be 'converted' into a MBS. At this stage, let us note some 'strange' features of $M_{2}$. First, in $M_{2}$ a point above some two choice points is always above an infinite number of choice points. Also, in $M_{2}$ one can define a certain 'odd' subset $X$, of which our Postulate B (to be introduced later) is true. Namely,

$$
X:=\left\{\left\langle\frac{3}{2}, n, 0\right\rangle \mid n \in \mathcal{N}\right\}
$$

$X$ is strange because it is not a subset of any history, yet, every finite subset of $X$ is contained in some history.

\subsection{Results}

One might expect that there are cases of INFFB that involve FINFB: indeed the theorem below justifies this intuition and gives it a precise reading.

Theorem 15 If $\langle S, f\rangle$ is a case of FINFB and for some history $h_{S}: S \subseteq h_{S}$, then there are $S^{\prime} \subseteq W$ and $f^{\prime} \in \prod_{e \in S^{\prime}} \prod_{e}$ such that $\left\langle S^{\prime}, f^{\prime}\right\rangle$ is a case of INFFB.

Proof: By the assumption, there are $A, B \subseteq S$ such that (1) $A S L R B$ and (2) $h_{A} \in \bigcap\{f(e): e \in A\},(3) h_{B} \in \bigcap\{f(e): e \in B\}$ but (4) $\{\{f(e): e \in$ $A \cup B\}=\emptyset$. Clearly, (5) $A \subseteq h_{A} \cap h_{S}$ and (6) $B \subseteq h_{B} \cap h_{S}$. If $A \cup B$ is infinite, put: $S^{\prime}=A \cup B$ and $f^{\prime}=f_{\mid A \cup B}$, and go to (†). If $A \cup B$ is is finite, define now $S^{\prime}:=\left\{x \in h_{S} \mid \neg \exists e_{1} \in A, e_{2} \in B\left(x>e_{1} \wedge x>e_{2}\right)\right\}$.

Let us first argue that $S^{\prime}$ is infinite. Pick $a$ : a maximal element of $A$ and $b$ : a maximal element of $B$; since both share $h_{S}$, there is $y$ such that $y>a \wedge y>b$. Consider next a chain $l_{a}$ from $y$ to $a$, such that $\inf \left(l_{a}\right)=a$. If (7) $\forall x(x>a \rightarrow x>b)$, then $l_{a}$ is lower bounded by $b$. Yet, since $a$ is the infimum, i.e., the greatest lower bound of $l_{a}$, it must be that $a \geqslant b$, which contradicts (1). Hence, since (7) cannot be true, there is an $x$ such that $x<y$ and $x>a$ but $x \ngtr b$. BST's postulate of density implies that $Z:=\left\{z \in l_{a} \mid z<x\right\}$ is infinite; since $Z \subseteq S^{\prime}, S^{\prime}$ is infinite as well.

As for the next condition of INFFB, since $S^{\prime} \subseteq h_{S}$, it must be that $\forall A \subseteq$ fin $S^{\prime} A \subseteq h_{S}$. 
We define $f^{\prime} \in \prod_{e \in S^{\prime}} \prod_{e}$ :

$$
f^{\prime}(e)=\left\{\begin{array}{l}
f(e) \text { if } e \in A \cup B \\
\Pi_{e}\left\langle h_{A}\right\rangle \text { if } e \notin A \cup B \text { and } \exists x \in A(e<x) \\
\Pi_{e}\left\langle h_{B}\right\rangle \text { if } e \notin A \cup B \text { and } \exists x \in B(e<x) \\
\Pi_{e}\left\langle h_{S}\right\rangle \text { otherwise }
\end{array}\right.
$$

$(\dagger)$ By the definitions of $S^{\prime}$ and $f^{\prime}$ and (1)-(6) we have: $\forall e, e^{\prime} \in S^{\prime}\left(e<e^{\prime} \rightarrow\right.$ $\left.f^{\prime}\left(e^{\prime}\right) \subseteq f^{\prime}(e)\right)$. By (4), since $A \cup B \subseteq S^{\prime}$, we get $\bigcap\left\{f^{\prime}(e): e \in S^{\prime}\right\}=\emptyset$.

Our title question, however, is just the opposite: are there cases of INFFB that do not involve FINFB? Before we turn to our main theorems, let us first show some simpler facts:

Corollary 16 Suppose that $A \subseteq S$ is finite and pairwise $S L R$. Then, if $S$ does not give rise to FINFB, $\bigcap_{e \in A}\{f(e)\} \neq \emptyset$ for any $f \in \prod_{e \in S} \prod_{e}$.

The corollary stems from the fact that any finite set is a union of a finite family of singletons.

Theorem 17 Assume that $S$ is an infinite set of pairwise SLR points such that for some history $h, S \subseteq h$.

If there exist sets $A_{1}, A_{2}$ such that $A_{1} \cup A_{2}=S$ and none of them gives rise to INFFB, then (if $S$ gives rise to INFFB, then $S$ gives rise to FINFB).

Proof: From the first antecedent we get that $\forall f \in \prod_{e \in A_{1}}: \bigcap\{f(e): e \in$ $\left.A_{1}\right\} \neq \emptyset$ and a similar result for $A_{2}$. From the second antecedent we get that $\exists g \in \prod_{e \in S}: \bigcap\{f(e): e \in S\}=\emptyset$. We can of course think of the function $g$ defined on $S$ as a union of two functions defined respectively on $A_{1}$ and $A_{2}$. Thus, we see that $\langle S, g\rangle$ constitute a case of FINFB because $\bigcap\{g(e)$ : $\left.e \in A_{i}\right\} \neq \emptyset$ and $\bigcap\left\{g(e): e \in A_{2}\right\} \neq \emptyset$ while $\bigcap\left\{g(e): e \in A_{1} \cup A_{2}\right\}=\emptyset$. Therefore $S$ gives rise to FINFB. Q.E.D.

The above theorem yields us the following simple corollary:

Corollary 18 Assume $S$ is an infinite set of pairwise SLR points such that for some history $h, S \subseteq h$. Then, if $S$ does not give rise to FINFB and there exists a cofinite subset of $S$ which does not give rise to INFFB, then the whole set $S$ does not give rise to INFFB. 
Turning to our main theorems characterizing INFFB, we will now introduce two postulates and prove a few theorems about and show how they relate to FINFB and INFFB.

Postulate 19 (Postulate $\boldsymbol{A}$ ) There exist 1) a set $S \subseteq W$ which is an infinite set of pairwise SLR points such that for some history $h S \subseteq h$ and 2) a function $f \in \prod_{e \in S} \prod_{e}$ such that

$\exists e \in S \forall h \in$ Hist $\forall x \in W:$

$$
\left(x \notin h \vee \neg(x>e) \vee h \notin f(e) \vee \exists e_{1} \in S\left(h \notin f\left(e_{1}\right) \wedge \neg\left(x S L R e_{1}\right)\right)\right)
$$

The motivation for this postulate comes from a certain structure, called $M_{1}$, that Müller et al. (2006) introduced. In this structure, one tries 'by hand' to prohibit the existence of a certain history, by this means producing a case of INFFB, without there being a case of FINFB. This procedure, however, fails if Postulate $\mathrm{A}$ is false. In this case, a seemingly excluded history gets re-inserted "by force" by Kuratowski-Zorn Lemma. Namely, the falsity of Postulate A ensures the existence of a certain function that can be used to produce a chain of events, which extends, by Kuratowski-Zorn lemma to the seemingly excluded history. More precisely, if Postulate A is false, then for any infinite pairwise SLR set $S$ such that for some history $h S \subseteq h$ and for any function $f \in \prod_{e \in S} \prod_{e}$ we can define a function $F: S \rightarrow H i s t \times W$ in the following way $(e \in S)$ :

$$
F(e):=\langle h, x\rangle:\left(x>e \wedge x \in h \wedge h \in f(e) \wedge \forall_{e^{\prime} \in S}\left(h \notin f\left(e^{\prime}\right) \Rightarrow e^{\prime} S L R x\right)\right)
$$

(Of course many different functions meeting this requirement might exist as there might be many equally good candidates for $h$ and $x$ such that for a given $e F(e)=\langle h, x\rangle$. What is important for us is that, when Postulate $\mathrm{A}$ is false, such functions do exist; we will just choose one.) Thus, we assume Postulate A in order Kuratowski-Zorn lemma not to produce unwanted histories.

As for the second postulate, it relates to structure $M_{2}$ and a question why it contains a case of INFFB. Our tentative diagnosis is that in $M_{2}$ one can define a certain 'odd' subset $X$ (see Equation 18), of which the postulate below is true:

Postulate 20 (Postulate $\boldsymbol{B}$ ) There is a set $X \subseteq W$ such that

(a) for any $A \subseteq$ fin $X$, there is a history $h: A \subseteq h$; 
(b) there is no history $h$ such that $X \subseteq h$.

The theorems we will show are summarized in the list below:

1. (Theorem 21) PostulateA $\Rightarrow \mathrm{INFFB}$

2. (Theorem 22) Postulate $B \wedge$ NO FINFB $\Rightarrow$ INFFB

3. (Theorem 23) Given that the BST model has space-time points, NO FINFB $\wedge \neg($ Post.A $) \wedge \neg($ Post.B $) \Rightarrow$ NO INFFB

4. (Theorem 24) Postulate $A \nRightarrow$ FINFB

5. (Theorem 25) $\neg($ Postulate $A) \wedge$ Postulate $B \nRightarrow$ FINFB

As for "space-time points" mentioned in theorem 23, in its proof we want to be able to say that something happens "in the same space-time point" in different histories. A triple $\langle W, \leqslant, s\rangle$ is a "BST model with space-time points" $(\mathrm{BST}+\mathrm{S})$ iff $\langle W, \leqslant\rangle$ is a BST model and $s$ (from the expression "space-time point") is an equivalence relation on $W$ such that 1) for each history $h$ in $W$ and for each equivalence class $s(x), x \in W$, the intersection $h \cap s(x)$ contains exactly one element and 2) $s$ respects the ordering: for equivalence classes $s(x), s(y)$ and histories $h_{1}, h_{2}, s(x) \cap h_{1}=s(y) \cap h_{1}$ iff $s(x) \cap h_{2}=s(y) \cap h_{2}$, and the same for " $<$ " and " $>$ ". As Müller shows in Müller (2005), not every BST model can be extended to a BST $+\mathrm{S}$ model, so our theorem is not as general as we would ideally prefer.

Theorem 21 Suppose Postulate $A$ is true due to some $S \subseteq W$ and $f \in$ $\prod_{e \in S} \Pi_{e}$. Then $\langle S, f\rangle$ is a case of INFFB.

Proof: Since by the assumption $S$ is infinite, pairwise SLR, and for some history $h$ : $S \subseteq h$, we have: (1) $\operatorname{card}(S) \geqslant \omega$ and (2) $\forall A(A \subseteq$ fin $S \rightarrow \exists h \in$ Hist: $A \subseteq h)$, and (3) $\forall e, e^{\prime} \in S\left(e<e^{\prime} \rightarrow f\left(e^{\prime}\right) \subseteq f(e)\right)$. We need thus to show (4) $\bigcap\{f(e) \mid e \in S\}=\emptyset$. For reductio assume $\bigcap\{f(e) \mid e \in S\} \neq \emptyset$. Hence, there must be a history (a) $h^{*} \in \bigcap\{f(e) \mid e \in S\}$. Suppose $e^{*} \in S$ is one of the points of which the existential formula of Postulate A is true. Since it follows that $h^{*} \in f\left(e^{*}\right)$, it is true for $e^{*}$ that

$$
\forall x \in W\left(x \notin h^{*} \vee \neg\left(x>e^{*}\right) \vee \exists e_{1} \in S\left(h^{*} \notin f\left(e_{1}\right) \wedge \neg\left(x S L R e_{1}\right)\right)\right) .
$$

Again, since $h^{*} \in f\left(e^{*}\right)$ and there are no maximal elements in the model (see BST postulate 2 of Definition 2), we can find a point $x^{*}$ such that $x^{*}>e^{*}$ and $x^{*} \in h^{*}$. In other words, for this $x^{*}$ two elements of the above alternative 
are false - so the third one must be true. But it also is false, since one of the conjuncts is always false: namely, because of (a) it can't be true for any $e_{1} \in S$ that $h^{*} \notin f\left(e_{1}\right)$. So the whole alternative is false for $x^{*}$, and thus we arrive at a contradiction. Therefore $\bigcap\{f(e) \mid e \in S\}=\emptyset$ so $\langle S, f\rangle$ constitute a case of INFFB. Q.E.D.

We will now establish that INFFB follows from Postulate B together with NO FINFB. Suppose that Postulate B is true due to a certain set $X$. Our goal is to find a set $S$ and a function $f$ such that $\langle S, f\rangle$ is a case of INFFB.

Theorem 22 Suppose that in a BST model $\mathcal{W}=\langle W, \leqslant\rangle$ Postulate $B$ is true and no $S \subseteq W$ gives rise to FINFB. Then there exists a case of INFFB in the model, i.e., there exists a set $S \subseteq W$ and a function $f \in \prod_{e \in S} \prod_{e}$ such that $\langle S, f\rangle$ is a case of INFFB.

Proof: Let $X \subseteq W$ be a set of which Postulate B is true. Define:

$$
\begin{gathered}
C(x):=\left\{e \in W \mid \exists h\left(h \in H i s t \wedge h \perp_{e} H_{(x)}\right)\right\} \\
S:=\bigcup_{x \in X} C(x)
\end{gathered}
$$

The assumption of NO FINFB and a fact about location of choice points (see Appendix, Fact 41) entail (a) $\forall e(e \in C(x) \rightarrow e<x)$, so it makes sense to write $\Pi_{e}\langle x\rangle$ if $e \in C(x)$. We thus tentatively define the product function $f$ on $S$ :

$f(e):=\Pi_{e}\langle x\rangle$ iff $e \in C(x)$.

To check that this is indeed a good definition, we need to prove that if (b) $e \in C(x) \cap C(y)$ and $x \neq y$, then $\Pi_{e}\langle x\rangle=\Pi_{e}\langle y\rangle$. Assume to the contrary that (c) $\Pi_{e}\langle x\rangle \neq \Pi_{e}\langle y\rangle$. Then, since by (a) and (b): $e<x \wedge e<y$, it must be by (c) that (d) $H_{(x)} \perp_{e} H_{(y)}$. But, by Postulate B (a), there must be a history $h^{*}$ such that $\{x, y\} \subseteq h$, and hence $h^{*} \in H_{(x)}$ and $h^{*} \in H_{(y)}$, which contradicts (d).

Turning to the conditions of INFFB, we will first argue that

(f) $\forall e, e^{\prime} \in S\left(e<e^{\prime} \rightarrow f\left(e^{\prime}\right) \subseteq f(e)\right)$.

If $e, e^{\prime} \in C(x)$, then by the definition of $f: f(e)=f\left(e^{\prime}\right)$. So, let $e \in C(x)$, $e^{\prime} \in C(y)$ and $x \neq y$. By Postulate B (a), $\exists h_{x y} \in H:\{x, y\} \subseteq h_{x y}$. Accordingly, $h_{x y} \in \Pi_{e}\left\langle h_{x y}\right\rangle=\Pi_{e}\langle x\rangle=f(e)$ and $h_{x y} \in \Pi_{e^{\prime}}\left\langle h_{x y}\right\rangle=\Pi_{e^{\prime}}\langle y\rangle=$ $f\left(e^{\prime}\right)$. Accordingly, $\Pi_{e}\langle x\rangle \cap \Pi_{e^{\prime}}\langle y\rangle \neq \emptyset$. Moreover, since $e<e^{\prime}, \Pi_{e^{\prime}}\langle y\rangle \subseteq$ $\Pi_{e}\langle x\rangle \neq \emptyset$, i.e., $f\left(e^{\prime}\right) \subseteq f(e)$.

Next, we prove the following:

(g) If $h \in \bigcap\{f(e) \mid e \in S\}$, then $X \subseteq h$.

Indirectly, let for some $h: h \in \bigcap\{f(e) \mid e \in S\}$ and assume that there is 
$x \in X$ such that $x \notin X$. Take any $h_{x}$ such that $x \in h_{x}$. Clearly, $x \in h_{x} / h$, so by PCP: $\exists e \in W:\left(h \perp_{e} h_{x} \wedge e<x\right)$, from which (i) $h \perp_{e} H_{(x)}$ follows. Thus, $e \in C(x)$. By the assumption, $h \in f(e)=\Pi_{e}\langle x\rangle$, and hence $h \equiv_{e} H_{(x)}$. This contradicts (i), however.

Now, Postulate B (b) says that $\neg \exists h \in$ Hist $X \subseteq h$, so (g) implies that

(j) $\bigcap\{f(e) \mid e \in S\}=\emptyset$.

We next prove that

(k) if $A \subseteq_{\text {fin }} S$, then $\bigcap\{f(e) \mid e \in A\} \neq \emptyset$.

If $A$ is finite, then there is a set $A^{*}$ of maximal elements of $A$. Clearly, $A^{*}$ is pairwise SLR. Hence, since no $S \subseteq W$ gives rise to FINFB, it follows by Corollary (16) that

(l) $\bigcap\left\{f(e) \mid e \in A^{*}\right\} \neq \emptyset$. By the construction, if $e \in A / A^{*}$, then there is $e^{\prime} \in A^{*}$ such that $e<e^{\prime}$. By (f) then, $f\left(e^{\prime}\right) \subseteq f(e)$. This and (l) entail that $\bigcap\{f(e) \mid e \in A\} \neq \emptyset$.

Importantly, (k) has two sought-for consequences:

(m) if $A \subseteq$ fin $S$, then $\exists h \in H i s t(A \subseteq h)$ and

(n) $\operatorname{card}(S) \geqslant \omega$. Otherwise, by $(\mathrm{g})$ and $(\mathrm{k})$ there would be a history $h$ such that $X \subseteq h$, which contradicts Postulate B (b).

To see that $\langle S, f\rangle$ is a case of INFFB, we need to show that $(1) \operatorname{card}(S) \geqslant$ $\omega$ and (2) $\forall A(A \subseteq$ fin $S \rightarrow \exists h \in$ Hist $: A \subseteq h)$, and (3) $\forall e, e^{\prime} \in S(e<$ $\left.e^{\prime} \rightarrow f\left(e^{\prime}\right) \subseteq f(e)\right)$ and $(4) \bigcap\{f(e): e \in S\}=\emptyset$. Yet, we already established these conditions: (1) is (n), (2) is (m), (3) is (f) and (4) is (j).

Note that $S$, as constructed in the proof above, gives rise to INFFB but it needs neither to be pairwise SLR, nor a subset of a history. Thus, it might be that funny business generated by Postulate B is even stranger than expected, as for instance $S$ might have no maximal elements. To secure a more familiar INFFB, i.e., such that $S$ is pairwise SLR and a subset of a history, we need another requirement, called Supplement, which refers to $S$ from the proof above:

Supplement Every chain in $S$ is upper bounded and for some $h^{*} \in$ Hist: $S \subseteq h^{*}$.

We leave it to the reader to show that if Postulate B and Supplement are true and no $S \subseteq W$ gives rise to FINFB, then there exists a set $S^{*}$ that is pairwise SLR and a subset of history and gives rise to INFFB. The set in question can be defined as:

$$
S^{*}=\left\{\sup _{h^{*}}(l) \mid l \text { is a maximal chain in } S\right\}
$$

where $S$ is defined in (22) and $h^{*}$ is the history to which Supplement refers. The sought-for product function $f^{\prime}$ on $S^{*}$ should be defined for such $e$ that 
$e \in S^{*} / S$ as well. Thus,

$$
f^{\prime}(e):=\left\{\begin{array}{l}
\Pi_{e}\langle x\rangle \text { iff } e \in C(x) \\
\Pi_{e}\left\langle h^{*}\right\rangle \text { iff } e \in S^{*} / S
\end{array}\right.
$$

It is now time to prove our main theorem.

Theorem 23 Suppose $\mathcal{W}=\langle W, \leqslant\rangle$ is a $B S T+S$ model and no $S \subseteq W$ gives rise to FINFB. Suppose further that both Postulates $A$ and $B$ are false in $\mathcal{W}$. Then no infinite set $S$ of pairwise SLR points such that for some history $h$ : $S \subseteq h$ gives rise to INFFB.

Proof: Suppose that $S$ is infinite, pairwise SLR and a subset of a history. Since $\langle S, f\rangle$ not being a case of INFFB is equivalent to the disjunction of four conditions, it suffices to to show that one of these conditions obtains. That is, we will prove that for any product function $f$ on $S,($ a) $\bigcap\{f(e): e \in S\} \neq \emptyset$.

Consider $S$ as naturally indexed by its cardinality. Since Postulate A is false, there is a function $F: S \rightarrow H i s t \times W$ defined as in (19). Take $e_{0} \in S$. For some $x_{0} \in W$ and $h_{0} \in H i$ st we have that $F\left(e_{0}\right)=\left\langle h_{0}, x_{0}\right\rangle$. Consider $S_{0}:=\left\{e \in S: h_{0} \in f(e) \wedge x_{0}>e\right\}$. If $S_{0}=S$, we have completed the proof and $h_{0}$ is the desired history, since then $\forall e \in S_{0}\left(h_{0} \in f(e)\right)$.

Otherwise, the construction guarantees that $x_{0} S L R\left(S / S_{0}\right)$. Namely, if $e \in S / S_{0}$ and $h_{0} \notin f(e)$, then by the definition of $F$ (see eq. (19)), $x_{0}$ SLR $e$. And, if (b) $h_{0} \in f(e)$, it cannot be that $e<x_{0}$, because then $e \in S_{0}$. It cannot be that $e>x_{0}$, either, since this implies $e>e_{0}$. By (b): $e \in h_{0}$, and from the definition of $F: x_{0} \in h_{0}$, and hence $x_{0}$ SLR $e$.

Take then a point from $S / S_{0}$ (say, a point $e_{i}$ such that $i$ is the minimal index in the set of indexes of points from $\left.S / S_{0}\right)$ and call it $e_{1}$. So, for some $x_{1}^{\prime} \in W$ and $h_{1}^{\prime} \in$ Hist we have that $F\left(e_{1}\right)=\left\langle h_{1}^{\prime}, x_{1}^{\prime}\right\rangle$. From NO FINFB (applied to the SLR set $\left\{x_{0}, e_{1}\right\}$ ) we get that $H_{\left(x_{0}\right)} \cap \Pi_{e_{1}}\left\langle h_{1}^{\prime}\right\rangle \neq \emptyset$ so there is a history $h_{1}$ belonging to the intersection. Clearly, $x_{0} \in h_{1}$. Since $\mathcal{W}$ is by assumption a BST $+\mathrm{S}$ model, we can take a point $x_{1}:=s\left(x_{1}^{\prime}\right) \cap h_{1}$. Accordingly, $\left\{x_{0}, x_{1}\right\} \subseteq h_{1}$. We define $\Sigma_{1}:=\left\{x_{0}, x_{1}\right\}$ and $H_{\Sigma_{1}}:=\{h \in$ Hist $\left.\mid \Sigma_{1} \subseteq h\right\}$. Take $S_{1}:=\left\{e \in S / S_{0}: h_{1} \in f(e) \wedge x_{1}>e\right\}$. On the occasion that $S=S_{0} \cup S_{1}$ we have completed the proof and $h_{1}$ is the desired history. For, we have that $\forall e\left(e \in S_{0} \cup S_{1} \rightarrow h_{1} \in f(e)\right)$. If $S \neq S_{0} \cup S_{1}$, we continue similarly with a point $e_{2} \in S /\left(S_{0} \cup S_{1}\right)$.

The above two steps should give us an idea of what to do while moving from $e_{k}$ to $e_{k+1}$. Suppose we finished the $k$-th step and accordingly we have set $S_{k}$, history $h_{k}$ and set $\Sigma_{k} \subseteq h_{k}$. If $S / \bigcup_{0 \leqslant i \leqslant k} S_{i} \neq \emptyset$, the theorem is not 
proved yet, so we take a point from $S / \bigcup_{0 \leqslant i \leqslant k} S_{i}$ and label it $e_{k+1}$. So, for some $x_{k+1}^{\prime} \in W$ and $h_{k+1}^{\prime} \in$ Hist we have that $F\left(e_{k+1}\right)=\left\langle h_{k+1}^{\prime}, x_{k+1}^{\prime}\right\rangle$. From NO FINFB (applied to set $\Sigma_{k} \cup\left\{e_{k+1}\right\}$ as $\Sigma_{k} \operatorname{SLR}\left\{e_{k+1}\right\}$ ) we get that $H_{\Sigma_{k}} \cap \prod_{e_{k+1}}\left\langle h_{k+1}^{\prime}\right\rangle \neq \emptyset$ so there is a history $h_{k+1}$ belonging to the intersection. Take $x_{k+1}:=s\left(x_{k+1}^{\prime}\right) \cap h_{k+1}$ and put $\Sigma_{k+1}=\Sigma_{k} \cup\left\{x_{k+1}\right\}$. Of course $\Sigma_{k+1} \subseteq$ $h_{k+1}$. Define $S_{k+1}:=\left\{e \in S / \bigcup_{0 \leqslant i \leqslant k} S_{i}: h_{k+1} \in f(e) \wedge x_{k+1}>e\right\}$. On the occasion that $S=\bigcup_{0 \leqslant i \leqslant k+1} S_{i}$ we have completed the proof and $h_{k+1}$ is the desired history. If not, we continue similarly with a point $e_{k+2} \in S / \bigcup_{0 \leqslant i \leqslant k+1} S_{i}$.

Let us now move to the limit case. Consider the set $\bigcup_{k<\omega} \Sigma_{k}$. It possesses the following properties:

- For every finite subset $A \subseteq$ fin $\bigcup_{k<\omega} \Sigma_{k}$ there exists a history $h: A \subseteq h$ (since it is finite, $A$ has to be a subset of $\Sigma_{k}$ for some $k<\omega$, and so $\left.A \subseteq h_{k}\right)$;

- It is infinite $\left(\right.$ since $\left.\forall_{i, j}\left(i \neq j \Rightarrow \Sigma_{i} \neq \Sigma_{j}\right)\right)$.

Therefore, the set is of the kind that Postulate B speaks about. Since we assumed its negation, we infer that there is a history $h^{*} \in H i$ ist such that $\bigcup_{k<\omega} \Sigma_{k} \subseteq h^{*}$. If $S=\bigcup_{k<\omega} S_{k}$, the theorem is proved and $h^{*}$ is the desired history.

Suppose however that $S / \bigcup_{k<\omega} S_{k} \neq \emptyset$. Take a point $e_{\omega} \in S-\bigcup_{k<\omega} S_{k}$. So, for some $x_{\omega}^{\prime} \in W, h_{\omega}^{\prime} \in H i s t$ it is so that $F\left(e_{\omega}\right)=\left\langle h_{\omega}^{\prime}, x_{\omega}^{\prime}\right\rangle$. Consider sets $A_{1}:=\left\{e_{i}: 0 \leqslant i<\omega\right\}$ and $A_{2}:=\left\{e_{\omega}\right\}$ From the construction it follows that there are histories $h^{*}$ and $h_{\omega}^{\prime}$ such that $h^{*} \in \bigcap_{e \in A_{1}} f(e)$ and $h_{\omega}^{\prime} \in \bigcap_{e \in A_{2}} f(e)$. So, by NO FINFB (applied to $\left.A_{1} \cup A_{2}\right), \bigcap_{e \leqslant \omega} f(e) \neq \emptyset$, so there is a history $h_{\omega}$ belonging to the intersection. Put $x_{\omega}:=s\left(x_{\omega}^{\prime}\right) \cap h_{\omega}$ and let $\Sigma_{\omega}:=\left\{x_{\omega}\right\} \cup$ $\left\{s(x) \cap h_{\omega}: x \in \bigcup_{k<\omega} \Sigma_{k}\right\}$. Let $S_{\omega}:=\left\{e \in S / \bigcup_{k<\omega} \Sigma_{k}: h_{\omega} \in f(e) \wedge x_{\omega}>e\right\}$.

If $S=\bigcup_{k \leqslant \omega} S_{k}$ we have completed the proof and $h_{\omega}$ is the desired history. If not, we continue similarly with points from $S / \bigcup_{k \leqslant \omega} S_{k}$. Since we have given instructions on what to do with e point $e_{i}$ whether $i$ is a limit number or not (the above case with $\omega$ is easily generalized), we are bound to arrive at a desirable history $h \in \bigcap\{f(e): e \in S\}$. Q.E.D.

The last two theorems in this section are to show that the first two theorems from the list above are not useless: since FINFB leads to INFFB, we 
need to make sure that neither Postulate A nor Postulate B yields FINFB.

Theorem 24 If a set $S \subseteq W$ is an infinite set of pairwise $S L R$ points such that for some history $h S \subseteq h$ and a function $f \in \prod_{e \in S} \prod_{e}$ satisfies this condition:

$$
\begin{aligned}
& \exists e \in S \forall h \in H i s t \forall x \in W: \\
& \quad x \notin h \vee \neg(x>e) \vee h \notin F(e) \vee \exists e_{1} \in S\left(h \notin f\left(e_{1}\right) \wedge \neg\left(x S L R e_{1}\right)\right)
\end{aligned}
$$

then it does not follow that $\langle S, f\rangle$ is a case of FINFB.

Proof sketch. Our example will take place in a MBS. Unfortunately, during the construction we have run into similar problems as with theorem 35: namely, we can present a proper set if we restrict ourselves to $\mathbb{R}^{2}$, while the $\mathbb{R}^{4}$ case involves an intuitive extension of our idea which unfortunately would be formally painful. Thus we will show the $\mathbb{R}^{2}$ case. The second coordinate is spatial. (By " $(a, b)$ " we will sometimes mean "a point in $\mathbb{R}^{2}$ " or "a segment of $\mathbb{R}$ ", but it will always be clear from the context.)

Let $S_{1}=\left\{(0, x) \in \mathbb{R}^{2}: x \in(0,1)\right\}$ be a dense segment of splitting points. Suppose all choice points generated by $S_{1}$ are binary and label one possibility "0" and the other "1". Assume that each scenario from $\Sigma$ corresponds to a history belonging to only a finite number of "0"-possibilities (in harmony with lemma 8 , there are no other histories). Put $B:=\left(\Sigma \times \mathbb{R}^{2}\right) / \equiv_{S}$.

The set of choice points generated by $S_{1}$ will be called $S . S=\left\{\left[(0, x)_{\sigma}\right]\right.$ : $x \in(0,1), \sigma \in \Sigma\}$. Consider a function $f \in \prod_{e \in S} \prod_{e}$ such that

$$
f\left(\left[(0, x)_{\sigma}\right]\right)= \begin{cases}1 & \text { if } x \geqslant 1 / 2 \\ 0 & \text { if } x<1 / 2\end{cases}
$$

The point that will make Postulate $\mathrm{A}$ true is $\left[(0,1 / 2)_{\sigma}\right]$. It is because it is true that

$\forall x \in W \forall h \in$ Hist :

$$
\left(x \in h \wedge h \in f\left(\left[(0,1 / 2)_{\sigma}\right]\right) \wedge x>\left[(0,1 / 2)_{\sigma}\right]\right) \Rightarrow \exists e_{1} \in S\left(h \notin f\left(e_{1}\right) x>e_{1}\right)
$$

which we arrive at by transforming Postulate A. And the above is true because any point above $\left[(0,1 / 2)_{\sigma}\right]$ is also above an infinite number of points $\left[(0, x)_{\sigma}\right]$ such that $x \in(0,1 / 2)$. Any history has to belong to the "1"possibility in some of those points, contrary to what function $f$ dictates. Now we have to show that $\langle S, f\rangle$ do not constitute a case of FINFB. Consider $A, B \subseteq S$. If $\left\{\left[(0, x)_{\sigma}\right]: x \in(0,1 / 2)\right\} \cap A$ is infinite or $\left\{\left[(0, x)_{\sigma}\right]: x \in\right.$ 
$(0,1 / 2)\} \cap B$ is infinite, then from our assumption about the histories in our model we infer that $\bigcap\{f(e): e \in A\}=\emptyset($ resp. $\bigcap\{f(e): e \in A\}=\emptyset)$, so the antecedent from the definition of NO FINFB is false. In the other case, if both $\left\{\left[(0, x)_{\sigma}\right]: x \in(0,1 / 2)\right\} \cap A$ and $\left\{\left[(0, x)_{\sigma}\right]: x \in(0,1 / 2)\right\} \cap B$ are finite, then $\left\{\left[(0, x)_{\sigma}\right]: x \in(0,1 / 2)\right\} \cap(A \cup B)$ is finite, therefore (again, by our assumption about the histories in the model $) \bigcap\{(e): e \in A \cup B\} \neq \emptyset$, so the consequent from the definition of NO FINFB is false. Therefore, $\langle S, f\rangle$ do not constitute a case of FINFB.

Theorem 25 Suppose Postulate $A$ is false, whereas Postulate $B$ is true in our model and $X$ is the set whose existence is entailed by Postulate B. It does not follow that $S$ gives rise to FINFB.

Proof by observation: $M_{2}$ provides us with an appropriate example (see section 4.1). Set $X$, as defined in Equation (18), is exactly of the kind required by Postulate B. As for Postulate A, it is false iff for every infinite $S$ that is pairwise SLR and a subset of a history and for every product function on $S$, there is a function $F$, as defined in Equation (19). Clearly, in $M_{2}$ there are plenty of infinite sets that are pairwise SLR and subsets of a history. Yet, as long as such a set does not contain a choice point, the construction of a function satisfying the conditions on $F$ is straightforward and we leave it to the reader. The only case requiring some attention is if a set described above contains a choice point. Observe that the set of choice points in $M_{2}$ is $S^{\prime}=\{\langle 1, n\rangle: n \in \mathbb{N}\}$. Thus, we need to say what value $F$ takes on elements of $S^{\prime} \cdot 8$

$$
F(\langle 1, n\rangle):=\langle g,\langle 3 / 2, n, i\rangle\rangle,
$$

where $i=0,1$ and $g$ is some function from $\mathbb{F}$ such that $g(n)=i$. It is easy to see that this function satisfies conditions on $F$ (19). Thus, since for every infinite $S$ that is pairwise SLR and a subset of a history and for every product function on $S$, there is a sought-for function $F$, Postulate A is false in $M_{2}$. Finally, as shown in Müller et al. (2006), there is no funny business in $M_{2}$.

The upshot of this section is this: generally, there are exactly two ways of producing INFFB, where there is no case of FINFB: by Postulate A and by Postulate B. In the next section, we will investigate if these postulates can be true in MBS.

\footnotetext{
${ }^{8}$ We can write this since there is one-to-one correspondence between $\mathrm{Hist}$ and $\mathbb{F}$ in $\mathrm{M}_{2}$.
} 


\section{$5 \quad$ Funny business in MBS}

In this section we ask under what conditions there could be cases of INFFB in MBS. Since (as we have seen) there are generally two ways of producing INFFB, namely by our Postulates A and B, we also ask what these postulates amount to in MBS's.

We begin with showing a simple fact, namely that under some conditions, there is NO INFFB in MBS. Imagine an infinite, pairwise SLR set of choice points. If you can divide the set into finite "chunks" separated by a minimal distance, the set does not give rise to infinitary funny business.

Condition 26 There exists a real number $\delta \in \mathbb{R}$ such that for any infinite, pairwise $S L R$ set of choice points $S$ and for any $x \in \mathbb{R}^{4}$, if $x$ is above only a finite number of points of $S_{M}$, then so is $\left\langle x^{0}+\delta, x^{1}, x^{2}, x^{3}\right\rangle \in \mathbb{R}^{4}$.

Notice that truth of condition 26 in a given model implies, for example, that there are no convergent sequences in any pairwise SLR set of choice points $\mathrm{S}$ in that model.

Fact 27 Suppose NOFINFB is true in an $M B S \mathcal{W}$. If condition [26 is also true in $W$, then no $\langle S, f\rangle$ such that $S$ is an infinite, pairwise SLR set of choice points and $f \in \prod_{e \in S} \prod_{e}$ gives rise to INFFB.

Proof: Suppose $S$ is an infinite, pairwise SLR set of choice points, $f \in \prod_{e \in S} \prod_{e}$ and $\langle S, f\rangle$ gives rise to $\operatorname{INFFB}\left({ }^{*}\right)$. We will obtain a contradiction by showing that there is a history $h_{L} \in \bigcap_{e \in S} f(e)$, and we will arrive at this history by constructing a certain denumerable chain $L=\left\{l_{0}, l_{1} \ldots\right\}$.

Take a point $\left[e_{0} \sigma\right] \in S 9$. Put $l_{0}:=\left[e_{0} \sigma\right]$ and $S_{0}:=\left\{\left[e_{0} \sigma\right]\right\}$. Since $e_{0}$ is above only a finite number of points of $S_{M}$ (one, to be exact), we apply condition 26 and put $x_{1}:=\left\langle e_{0}^{0}+\delta, e^{1} \cdot e^{2}, e^{3}\right\rangle$. Let $S_{1}=\left(S / S_{0}\right) \cap\left\{\left[x_{\sigma}\right]: x<_{M}\right.$ $x_{1}$ and $\sigma$ is a scenario $\} . S_{1}$ is finite. Therefore, by NOFINFB, both $\bigcap_{e \in S_{0}} f(e)$ and $\bigcap_{e \in S_{1}} f(e)$ are nonempty; so, again by NOFINFB, $\bigcap_{e \in S_{0} \cup S_{1}} f(e) \neq \emptyset$. We take a history $h_{\sigma_{1}} \in \bigcap_{e \in S_{0} \cup S_{1}} f(e)$ and put $l_{1}:=\left[x_{1} \sigma_{1}\right]$. It is evident from the construction that $l_{0}<l_{1}$.

\footnotetext{
${ }^{9}$ We hope it is clear from the context that $e_{0} \in \mathbb{R}^{4}$ and $\sigma \in \Sigma$. For clarity, in similar cases (when the point from $\mathbb{R}^{4}$ has a subscript) we do not want to write the scenario as a subscript, too.
} 
Suppose $l_{k}=\left[x_{k} \sigma_{k}\right]$. Since $x_{k}$ is above only a finite number of points of $S_{M}$ (they all belong to the set $\bigcup_{i=0}^{k} S_{i}$, which is a sum of a finite number of finite sets), we apply condition $\left[26\right.$ and put $x_{k+1}:=\left\langle x_{k}^{0}+\delta, e^{1} \cdot e^{2}, e^{3}\right\rangle$. Let $S_{k+1}=\left(S / \bigcup_{i=0}^{k} S_{i}\right) \cap\left\{\left[x_{\sigma}\right]: x<_{M} x_{k+1}\right.$ and $\sigma$ is a scenario $\} . S_{k+1}$ is finite. Then $\bigcap_{e \in \bigcup_{i=0} S_{i}} f(e) \neq \emptyset$ (because e.g. $h_{\sigma_{k}}$ belongs to it) and $\bigcap_{e \in S_{k+1}} f(e) \neq \emptyset$

(by NOFINFB). Then, again by NOFINFB, $\bigcap_{k+1} f(e) \neq \emptyset$. We take a $e \in \bigcup_{i=0}^{k+1} S_{i}$

history $h_{\sigma_{k+1}} \in \bigcap_{e \in \bigcup_{i=0}^{k+1} S_{i}} f(e)$ and put $l_{k+1}:=\left[x_{k+1} \sigma_{k+1}\right]$. It is evident from the construction that $l_{k}<l_{k+1}$.

We have described the procedure for creating the chain $L$. By laws of BST, there has to be a history $h_{L}$ containing the chain. We claim that $h_{L} \in \bigcap_{e \in S} f(e)$. Suppose, to the contrary, that $h_{L} \notin \bigcap_{e \in S} f(e)$. So $\exists e \in S$ : $h_{L} \notin f(e)(* *)$. By our construction, there has to be a natural $n$ such that $e \in S_{n}$. Since $h_{L} \equiv_{e} h_{\sigma_{n}}$ and $h_{\sigma_{n}} \in f(e)$, we get that $h_{L} \in f(e)$, which contradicts $\left(^{* *}\right)$. We know, then, that $h_{L} \in \bigcap_{e \in S} f(e)$, which contradicts $\left(^{*}\right)$. Q.E.D.

We know now that under some conditions there is NO INFFB in an MBS. Since one way of introducing INFFB is via Postulate A, let us investigate what this postulate amounts to in an MBS. It will turn out that we can introduce a certain kind of funny business ("epsilon funny business", labeled $\epsilon F B$ ), present whenever Postulate $\mathrm{A}$ is true. This will give us some details about the situations in which funny business can arise in MBS'.

Definition 28 Let $S \subseteq W$. We will say that $S_{M}=\left\{z \in \mathbb{R}^{4}: \exists \sigma \in \Sigma\right.$ : $\left.\left[z_{\sigma}\right] \in S\right\}$ is a reduced set derived from $S$.

In other words, the reduced set of a subset $S$ of $W$ is what we get after projecting $S$ on $\mathbb{R}^{4}$; it is the set of spatiotemporal locations occupied by members of $S$.

Definition 29 We say that in an MBS model $\mathcal{W}$ an infinite pairwise SLR set $S$, whose all elements have the form $\left[z_{\sigma}\right]$ for a given $\sigma \in \Sigma$, and a product function $f$ constitute $\epsilon F B$ iff 


$$
\exists e^{*} \in S_{M} \forall \epsilon\left(e^{*}\right) \bigcap_{e \in \epsilon\left(e^{*}\right)} f([e \sigma])=\emptyset,
$$

where $S_{M}$ is the reduced set derived from $S, \epsilon\left(e^{*}\right)=\left\{e \in S_{M} \mid d\left(e, e^{*}\right)<\delta\right\}$ for some $\delta \in \Re$ and $d()$ is the Euclidean distance.

Theorem 30 If in an MBS model $\mathcal{W}$ there is (1) an infinite pairwise SLR set $S=\left\{\left[z_{\sigma}\right]: z \in Z \subset \Re^{4}\right\}$ and (2) a product function $f$ such that $S$ and $f$ constitute $\epsilon F B$, then Postulate $A$ is true in $\mathcal{W}$.

Proof: To prove Postulate A, take $S, f$ and some $e^{*}$, whose existence is postulated by $\epsilon \mathrm{FB}$. As a sidenote, observe that, since No FIN FB is assumed to be true, it follows from $\forall \epsilon\left(e^{*}\right) \bigcap_{e \in \epsilon\left(e^{*}\right)} f\left(\left[e_{\sigma}\right]\right)=\emptyset$ that every neighborhood $\epsilon\left(e^{*}\right)$ comprises infinitely many elements of $S_{M}$. This is possible only if $e^{*}$ is a point of convergence of a sequence in $S_{M}$ (a special case is dense $S_{M}$ ).

We need to show that for every triple $\langle\gamma, x, \alpha\rangle\left(\gamma, \alpha \in \Sigma, x \in \Re^{4}\right)$ the following formula is true:

$$
\begin{array}{r}
{\left[x_{\alpha}\right] \neq\left[x_{\gamma}\right] \vee\left[e_{\sigma}^{*}\right] \neq\left[e_{\alpha}^{*}\right] \vee x \ngtr e^{*} \vee \gamma \notin f\left(\left[e_{\sigma}^{*}\right]\right) \vee} \\
\exists\left[e_{\sigma}^{\prime}\right] \in S\left(\gamma \notin f\left(\left[e_{\sigma}^{\prime}\right]\right) \wedge \neg\left(x \operatorname{SLR}_{M} e^{\prime}\right)\right) .
\end{array}
$$

The formula is the disjunction $D_{1} \vee D_{2} \vee D_{3} \vee D_{4} \vee D_{5}$. For a triple $\langle\gamma, x, \alpha\rangle$ we consider now two cases. It may be that for that triple $D_{1} \vee D_{2} \vee D_{3} \vee D_{4}$ is true, and hence, obviously, the entire disjunction (23) is true as well. In the other case, $D_{1} \vee D_{2} \vee D_{3} \vee D_{4}$ is not true for the triple, i.e., the conjunction $\neg D_{1} \wedge \neg D_{2} \wedge \neg D_{3} \wedge \neg D_{4}$ obtains. For Postulate A to be true, in this case the triple must make $D_{5}$ true, i.e.,

$$
\exists\left[e_{\sigma}^{\prime}\right] \in S\left(\gamma \notin f\left(\left[e_{\sigma}^{\prime}\right]\right) \wedge \neg\left(x \operatorname{SLR}_{M} e^{\prime}\right)\right) .
$$

We will now show this. Since by $\neg D_{3}: x>e^{*}$, we may define a nonempty subset $B(x)$ of $S_{M}$, whose elements are in the backward light cone of $x$, i.e., $B(x):=\left\{z \in S_{M} \mid z<_{M} x\right\}$. Observe now that for all $e$ from $S_{M}$ (a) $e \in B(x)$ iff $\neg\left(e \operatorname{SLR}_{M} x\right)$. The implication to the right is obvious; to see that the reverse implication holds as well, note that it holds if $e^{\prime}<x$. And $e^{\prime}>x$ is impossible, since it implies (as $x>e^{*}$ ) that for some $e \in S_{M} e^{\prime}>e$, which contradicts that $S_{M}$ is pairwise SLR.

Now by (a), Formula 24 is equivalent to:

$$
\exists\left[e_{\sigma}^{\prime}\right] \in S\left(\gamma \notin f\left(\left[e_{\sigma}^{\prime}\right]\right) \wedge e^{\prime} \in B(x)\right),
$$


which is equivalent to:

$$
\neg \forall\left[e_{\sigma}^{\prime}\right] \in S\left(e^{\prime} \in B(x) \rightarrow \gamma \in f\left(\left[e_{\sigma}^{\prime}\right]\right)\right),
$$

and further equivalent to:

$$
\gamma \notin \bigcap_{e^{\prime} \in B(x)} f\left(\left[e_{\sigma}^{\prime}\right]\right) .
$$

From the construction of $B(x)$ and $\epsilon \mathrm{FB}$ :

$$
\exists \epsilon\left(e^{*}\right) \epsilon\left(e^{*}\right) \subseteq B(x) \wedge \bigcap_{e^{\prime} \in \epsilon\left(e^{*}\right)} f\left(\left[e_{\sigma}^{\prime}\right]\right)=\emptyset .
$$

It follows that $\bigcap_{e^{\prime} \in B(x)} f\left(\left[e_{\sigma}^{\prime}\right]\right)=\emptyset$. So it cannot be that $\gamma \in \bigcap_{e^{\prime} \in B(x)} f\left(\left[e_{\sigma}^{\prime}\right]\right)$, and thus the formula 25 and hence $D_{5}$ must be true.

\section{Q.E.D.}

Theorem 31 If Postulate $A$ is true in an MBS model $\mathcal{W}$, then there is in $\mathcal{W}$ an infinite pairwise $S L R$ set $S$, whose all elements have the form $\left[z_{\sigma}\right]$ for a given $\sigma \in \Sigma$, and a product function $f$ such that $S$ and $f$ constitute $\epsilon F B$.

Proof: Let Postulate A be true in $\mathcal{W}$ due to $S, f$, and $e^{*}$. For reductio, assume that $S$ and $f$ do not constitute $\epsilon \mathrm{FB}$. We get that $\exists \epsilon\left(e^{*}\right) \bigcap_{e \in \epsilon\left(e^{*}\right)} f\left(\left[e_{\sigma}\right]\right) \neq$ $\emptyset$. We will construct a triple $x, \gamma, \alpha$ that contradicts Postulate A. Take the $\gamma \in \bigcap_{e \in \epsilon\left(e^{*}\right)} f\left(\left[e_{\sigma}\right]\right)$ for some $\epsilon\left(e^{*}\right)$ and $x>_{M} e^{*}$ such that $B(x) \subseteq \epsilon\left(e^{*}\right)$. (It is always possible to find such an $x$ since we are in an MBS and thus are searching in $\mathbb{R}^{4}$.) Accordingly, $\gamma \in \bigcap_{e \in B(x)} f\left(\left[e_{\sigma}\right]\right)$. Because $\forall e^{\prime}\left(e^{\prime} \in S / B(x) \rightarrow\right.$ $\left.x S L R_{M} e^{\prime}\right)$, we arrive at a contradiction with $D_{5}$. Since clearly $e^{*} \in \epsilon\left(e^{*}\right)$, we have $\gamma \in f\left(\left[e_{\sigma}^{*}\right]\right)$, which contradicts $D_{4} . x>_{M} e^{*}$ contradicts $D_{3}$. To obtain a contradiction with $D_{1}$, let $\alpha=\gamma$. Since $\gamma \in f\left(\left[e_{\sigma}^{*}\right]\right),\left[e_{\sigma}^{*}\right]=\left[e_{\gamma}^{*}\right]$, and hence $\left[e_{\sigma}^{*}\right]=\left[e_{\alpha}^{*}\right]$, which contradicts $D_{2}$. Thus, the triple $x, \alpha, \gamma$ contradicts the disjunction $D_{1} \vee D_{2} \vee D_{3} \vee D_{4} \vee D_{5}$, and hence Postulate A. Q.E.D.

Theorems 30 and 31 give us the following fact about the equivalence of Postulate A and epsilon funny business:

Fact 32 Let $\mathcal{W}$ be an MBS model. The following conditions are equivalent:

(1) Postulate $A$ is true in $\mathcal{W}$

(2) There is in $\mathcal{W}$ an infinite pairwise $S L R$ set $S=\left\{\left[z_{\sigma}\right]: z \in S_{M} \subseteq \Re^{4}\right\}$ and a product function $f$ such that $S$ and $f$ constitute $\epsilon F B$. 
Note the consequences of this fact. In order Postulate A be true in an MBS, there must be in the reduced set $S_{M}$ such an $e^{*}$ that $\bigcap_{e \in \epsilon\left(e^{*}\right)} f\left(\left[e_{\sigma}\right]\right)=\emptyset$, no matter how small the diameter of $\epsilon\left(e^{*}\right)$ is. The intersection cannot be empty if it is possible to have an $\epsilon\left(e^{*}\right)$ to which only $e^{*}$ belongs. Also, on the supposition that NO FINFB is true, the intersection cannot be empty if some $\epsilon\left(e^{*}\right)$ contain only a finite subset of $S_{M}$. Hence, in MBS Postulate A can be true provided that $S_{M}$ contain a convergent sequence, together with its point of convergence.

We will finally check whether Postulate B is false in MBS'. To this end, it would be enough to prove that in an MBS, if $\neg$ Postulate A and NO FINFB were true, then NO INFFB was true as well. Given the Theorem (22), this would imply that Postulate B was false in MBS'.

To begin our exploration, let us ask what form a pairwise SLR set $S$, which is a subset of a history, should take to give rise to funny business, INFFB or FINFB, in an MBS. Assume thus that $S$ is a subset of $W$, all elements of which have the form $\left[e_{\sigma}\right]$ for a given $\sigma \in \Sigma$. Clearly, $S$ is a subset of a history. Consider a product function $f$ on $S$, and assume that $\langle S, f\rangle$ is a case of funny business, FINFB or INFFB. Thus,

$$
\bigcap_{\left[e_{\sigma}\right] \in S} f\left(\left[e_{\sigma}\right]\right)=\emptyset
$$

Pick now some $a^{*}=\left\langle a_{0}^{*}, a_{1}^{*}, a_{2}^{*}, a_{3}^{*}\right\rangle \in S_{M}$ and consider elements of $\Re^{4}$ located 'vertically' above $a^{*}$, i.e., the set $L:=\left\{x \mid x=\left\langle x_{0}, a_{1}^{*}, a_{2}^{*}, a_{3}^{*}\right\rangle \wedge x_{0} \geqslant a_{0}^{*}\right\}$.

Our guiding idea is this: we want to see what has to happen if $\langle S, f\rangle$ is to be a case of funny business, FINFB or INFFB. Usually there exist 'safe' subsets of $S$ such that there is a history passing through all the elementary possibilities designated by the function $f$ at the points from the subsets. Suppose that it is not the case regarding the whole set $S$ (meaning that we have an example of funny business). What, then, with the 'in-between' section - the sets larger then the obviously 'safe' ones and smaller than the whole $S$ ? Does there have to be a biggest 'safe' set, or a smaller 'unsafe' set? Let us put these ideas down formally. For $x \in L$, we will say that $x$ is good iff for $S_{x}:=\left\{\left[e_{\sigma}\right] \in S \mid e \leqslant_{M} x\right\}: \bigcap_{\left[e_{\sigma}\right] \in S_{x}} f\left(\left[e_{\sigma}\right]\right) \neq \emptyset$. If $x$ is not good, we will say it is bad.

Clearly, $a^{*}$ is good, and if $x, y \in L, x<_{M} y$, and $y$ is good, then $x$ is good as well. Hence there are initial non-empty segments of $L$, whose all elements are good. We call such initial segments "good". The question now is: what maximal good initial segments of $L$ are possible? (Since the sum of a set 
of good segments is a good segment, for any chain a maximal good initial segment will exist.) There are two possibilities:

(1) The maximal good initial segment of $L$ is not upper bounded, and hence is identical to $L$. By an argument similar to that given at the end of our proof of Fact 27, there is a history $h_{L} \in \bigcap_{\left[e_{\sigma}\right] \in S} f\left(\left[e_{\sigma}\right]\right)$; hence $\langle S, f\rangle$ is not a case of funny business, i.e., we have neither FINFB nor INFFB.

(2) The maximal good initial segment of $L$ is upper bounded, so by properties of $\Re^{4}$ and our construction, it has a supremum; dub it $x^{*}$. Is then $x^{*} \operatorname{good}$ or bad? Again, there are two options:

(2a) $x^{*}$ is bad. We already know that for any $x<x^{*}, x \in L$ is good. Hence, funny business is located, so to speak, on the backward light cone of $x^{*}$, i.e., on $B_{\mid x^{*}}=\left\{x \in \Re^{4} \mid x<_{M} x^{*} \wedge D_{M}^{2}\left(x, x^{*}\right)=0\right\}$ (where $D_{M}^{2}$ is the Lorentz interval). In other words, defining $S^{\prime}=\left\{\left[e_{\sigma}\right] \in S \mid e \in B_{\mid x^{*}}\right\}$, we have it that $\left\langle S^{\prime} f\right\rangle$ is a case of funny business, FINFB or INFFB.

(2b) $x^{*}$ is good. This means that any $x>x^{*}, x \in L$ is bad. Hence, on any outer lining (however thin) $B_{x^{*}}(\delta)=\left\{x \in \Re^{4} \mid x<_{M} x^{*}(\delta) \wedge x \mathbb{x}_{M} x^{*}\right\}$, where $x^{*}(\delta)=\left\langle x_{0}^{*}+\delta, x_{1}^{*}, x_{2}^{*}, x_{3}^{*}\right\rangle$, of the backward light cone of $x^{*}$, there is located a case of funny business. More precisely, defining $S(\delta)=\left\{\left[e_{\sigma}\right] \in S \mid\right.$ $\left.e \in B_{x^{*}}(\delta)\right\}$, we have it that for any $\delta,\langle S(\delta), f\rangle$ is a case of funny business, FINFB or INFFB.

Thus, the result of this exploration is that if a pairwise SLR set $S$ (where $S \subseteq h$ for some history) gives rise to funny business in an MBS, then there is $x^{*} \in \Re^{4}$ and one of two conditions obtains:

(1) on the backward light cone of $x^{*}$ there is the reduced set $S_{M}^{\prime}$ of $S^{\prime}$ such that $S^{\prime} \subseteq S$ and $S^{\prime}$ gives rise to funny business, or

(2) on any outer lining of the backward light cone of $x^{*}$ there is the reduced set $S_{M}(\delta) \in \Re^{4}$ of $S(\delta)$ such that $S(\delta) \subseteq S$ and $S(\delta)$ gives rise to funny business. To put it more precisely,

Fact 33 If a pairwise SLR set $S$, which is a subset of a history gives rise to funny business (FINFB or INFFB) in an MBS, then there is $x^{*} \in \Re^{4}$ such that either (1) there is a set $S^{\prime} \subseteq S$ giving rise to funny business, whose reduced set $S_{M}^{\prime}$ is a subset of $B_{\mid x^{*}}=\left\{x \in \Re^{4} \mid x<_{M} x^{*} \wedge D_{M}^{2}\left(x, x^{*}\right)=0\right\}$, or (2) there is a family of sets $S(\delta) \subseteq S, \delta>0$, each $S(\delta)$ giving rise to funny business, and such that the reduced set $S_{M}(\delta)$ of $S(\delta)$ is a subset of $B_{x^{*}}(\delta)=$ $\left\{x \in \Re^{4} \mid x<_{M} x^{*}(\delta) \wedge x \nless_{M} x^{*}\right\}$, where $x^{*}(\delta)=\left\langle x_{0}^{*}+\delta, x_{1}^{*}, x_{2}^{*}, x_{3}^{*}\right\rangle$.

Note that $S$ can be different from $S^{\prime}$ as well as from any $S(\delta)$.

Let us observe the first consequence of this exploration: in MBS-like models produced on $\Re^{2}$, a pairwise SLR set which is a subset of a history 
cannot give rise to INFFB if $\neg$ Postulate A and NOFINFB are true 10 In the two dimensional case, the backward light cone $B_{\mid x^{*}}$ is reduced to two straight lines, call them "left" and "right". Since $S=\left\{\left[e_{\sigma}\right] \mid e \in S_{M}\right\}(\sigma \in \Sigma)$ is assumed to be pairwise SLR, $S_{M}$ could have at most two elements located on $B_{\mid x^{*}}$ (one of the left line, the other on the right line). Thus, in twodimensional case, if NOFINFB is true, there can be no INFFB located on the backward light cone of $x$. Can there be INFFB located on outer linings $B_{x^{*}}(\delta)$ ? Consider outer linings of one line, say left one. Such outer linings can be written as $B_{L}(\delta)=\left\{\left\langle y_{0}, y_{1}\right\rangle \in B_{x^{*}}(\delta) \mid y_{1}<x_{1}^{*}\right\}$. Note that, for $e \in S_{M}$, if $e \in B_{L}(\delta)$, then no $e^{\prime} \in S_{M}$ such that $e^{\prime}<_{M} e$ can belong to $B_{L}(\delta)$. In a similar vein, no $e^{\prime} \in S_{M}$ such that $e^{\prime}>_{M} a^{*}$ can belong to $B_{L}(\delta)$, where $a^{*}$ is that element of $S_{M}$ from which we started the construction of the chain $L$ containing $x^{*}$. Thus, $B_{L}(\delta)$ cannot go infinitely down along the left line, or up to $x$. Hence, given that NOFINFB is true, in order every $B_{L}(\delta)$ (i.e., for any $\delta>0$ ) generate INFFB, there must be a sequence of elements of $S_{M}$ converging to a point from the left line. Moreover, since elements of $S_{M}$ are $\mathrm{SLR}_{M}$, there must be exactly one point of convergence located on the left line. The same is true about right outer linings, $B_{R}(\delta)$. That is, for INFFB to obtain, a sequence in $S_{M}$ must converge to a point $e_{L}$ located on the left line or a sequence in $S_{M}$ must converge to a point $e_{R}$ located on the right line. Accordingly, we should consider three cases: (1) in $S_{M}$ there is only a sequence converging to a point $e_{L}$ on the left line, (2) in $S_{M}$ there is only a sequence converging to a point $e_{R}$ on the right line, and (3) in $S_{M}$ there are two sequences, one converging to a certain $e_{L}$ on the left line and the other converging to a certain $e_{R}$ on the right line. In each case we need to construct an outer lining $B_{x^{*}}\left(\delta^{*}\right)$ that does not generate INFFB. We will produce the required construction for case (3) only, as it is more complicated, and the reader will surely know how to transform it into arguments appropriate for the remaining two cases. Assume then case (3); it might happen that $e_{L}$ (or $e_{R}$ or both) does not belong to $S_{M}$. However, if $S_{M} \cup\left\{e_{L}, e_{R}\right\}:=S_{M}^{\prime}$ does not generate INFFB, $S_{M}$ does not generate it either. Consider thus the 'extended' set $S_{M}^{\prime}$. Assume $\neg$ Postulate A, which (as we have seen in Fact 32), is equivalent to NO $\epsilon$ FB. Apply then $\mathrm{NO} \epsilon \mathrm{FB}$ to $e_{L}$ and $e_{R}$, obtaining $\epsilon\left(e_{L}\right)$ and $\epsilon\left(e_{R}\right)$, estimated, resp., by diameters $\delta_{L}$ and $\delta_{R}$. Put $\delta^{*}=\min \left\{\delta_{L}, \delta_{R}\right\}$ and consider the outer lining $B_{x^{*}}\left(\delta^{*}\right)$. By our construction, the elements of $S_{M}$ that belong to $B_{x^{*}}\left(\delta^{*}\right)$, but neither to $\epsilon\left(e_{L}\right)$ nor to $\epsilon\left(e_{R}\right)$ constitute a finite set. By NO $\epsilon \mathrm{FB}$ we have,

\footnotetext{
${ }^{10}$ Strictly speaking, we defined MBS models as based on $\Re^{4}$, i.e., elements of our models are equivalence classes $\left[x_{\sigma}\right]$, where $x \in \Re^{4}$. There is no obstacle, however, to consider MBS-like models based on $\Re^{n}$ of different dimensionality.
} 
for any product function $f$ on $S^{\prime}=S \cup\left\{\left[e_{L} \sigma\right],\left[e_{R} \sigma\right]\right\}: \bigcap_{e \in \epsilon\left(e_{L}\right)} f\left(\left[e_{\sigma}\right]\right) \neq \emptyset$ and $\bigcap_{e \in \epsilon\left(e_{R}\right)} f\left(\left[e_{\sigma}\right]\right) \neq \emptyset$. Combining all these observations together, and assuming NOFINFB, we have an outer lining $B_{x^{*}}\left(\delta^{*}\right)$ that does not generate INFFB, i.e.

$\bigcap_{e \in S_{M} \cap B_{x^{*}}\left(\delta^{*}\right)} f\left(\left[e_{\sigma}\right]\right) \neq \emptyset$, which contradicts the result that every outer lining $B_{x^{*}}(\delta)$ generates INFFB.

One might hope that this result, i.e., in 2-dimensional MBS-like model, a pairwise SLR set $S$, which is a subset of a history cannot give rise to INFFB if NOFINFB and $\neg$ Postulate A are true, carries over to the real, i.e, 4-dimensional MBS. This hope is however shattered by a construction, which in essence consists in wrapping an $M_{2}$ structure around a backward light cone of some $x^{*}$.

Consider an MBS whose set $\Sigma$ of history labels is the set of all functions $g: \mathbb{N} \rightarrow\{0,1\}$ such that for only finitely many $n \in \mathbb{N}, g(n)=0$. Our construction of Section 3.1 guarantees that this is indeed an MBS, provided that the topological Postulate 6 is true, which we will check in a due course. Consider $x^{*}=\langle 0,0,0,0\rangle \in \Re^{4}$, its backward light cone $B_{\mid x^{*}}$, and a sequence of 'angles' $\varphi_{n}=\pi\left(2^{n}-1\right) / 2^{n}(n=1,2, \ldots)$. Let $S_{M}=\left\{\left\langle-n, n \cos \left(\varphi_{n}\right), n \sin \left(\varphi_{n}\right), 0\right\rangle \mid n=1,2, \ldots\right\}$. The (Euclidean) distance between any two elements of $S_{M}$ is at least $\sqrt{2}$. Also, $S_{M}$ is pairwise $\mathrm{SLR}_{M}$ and $S_{M} \subseteq B_{\mid x^{*}} . \quad e_{n} \in S_{M}$ belongs to appropriate sets of splitting points, according to this rule:

$$
\text { for } g, g^{\prime} \in \Sigma, e_{n} \in C_{g g^{\prime}} \text { iff } g(n) \neq g^{\prime}(n) \text {. }
$$

We can assign the same history label to all elements of $S_{M}$, for instance $g^{*}$ such that for all $n, g^{*}(n)=1$. Consider a product function $f$ on $S=$ $\left\{\left[e_{n} g^{*}\right] \mid e_{n} \in S_{M}\right\}$ defined as

$$
f\left(\left[e_{n} g^{*}\right]\right):=\{g \in \Sigma \mid g(n)=0\} .
$$

(Compare our discussion of elementary possibilities in $M_{2}$ on page 16.) Since among history labels there is no function $g$ that yields infinitely many zeros, $\langle S, f\rangle$ is a case of INFFB. On the other hand, $S$ does not give rise to FINFB, since there are history labels yielding arbitrarily large finite number of zeros. Also, since there is a minimal distance $\sqrt{2}$ between elements of $S_{M}$, one can associate with every $e_{n} \in S_{M}$ an $\epsilon\left(e_{n}\right)=\left\{e \in S_{M} \mid d\left(e, e_{n}\right)<\sqrt{2} / 2\right\}$ to which only the singleton $\left\{e_{n}\right\}$ belongs. The existence of such epsilons means that 
NO $\epsilon \mathrm{FB}$ is true in our model, and hence, by Fact 32, $\neg$ Postulate A is true as well. This means that Postulate B must be true in the model 11

Returning to the topological postulate, the only chains in $\Re^{4}$ that might falsify it are those that contain $x^{*}$. As an example, consider the vertical chain $L$ of elements below $x^{*}$, including $x^{*}$. Since histories are labeled by elements of the set $\Sigma$, we might write, for $z \in L, \Sigma_{g}(z):=\left\{g^{\prime} \in \Sigma \mid\left[z_{g}\right]=\left[z_{g^{\prime}}\right]\right\}$, like in Definition [5.

We have now, for every $y \neq x^{*} \in L: \Sigma_{g}(y)=\Sigma$, whereas $\Sigma_{g}\left(x^{*}\right)=\{g\}$. However odd it looks like, it means that our topological postulate is satisfied, and hence the described model is indeed an MBS. The oddity is similar to a feature of $M_{2}$ : no matter which history label is associated with $x^{*}$, the resulting event in BST is the smallest upper bound of some elements of $S$ and it cannot be SLR to any remaining element of $S$. The second trick of our MBS is that elements of $S_{M}$ escape steadily to the past, which ensures that there is always a finite distance between them. A natural question is whether $S_{M}$ bounded on temporal coordinates can yield INFFB if NO FINFB and $\neg$ Postulate A are true; we leave it as an open problem.

To sum up our investigations of MBS', we have the following:

(1) If NO FINFB and condition 26 are true, then no infinite and pairwise SLR set $S$ gives rise to INFFB.

(2) If in an MBS there is funny business (FINFB or INFFB) produced by a pairwise SLR subset $S$ of a history, then there is some $x \in \Re^{4}$ such that either (1) a set $S^{\prime} \subseteq S$, which gives rise to funny business and whose reduced set $S_{M}^{\prime}$ is located on the backward light cone of $x$, or (2) any outer lining of backward light cone of $x$ comprises the reduced set of a set $S(\delta) \subseteq S$ which generates funny business.

(3) In order for Postulate A to be true, given that NOFINFB obtains, there must be a set of choice points whose reduced set contains a converging sequence together with its point of convergence.

(4) Postulate B cannot be true in 2-dimensional MBS-like models if $\neg$ Postulate $\mathrm{A}$ and NOFINFB are true. However, there are 4-dimensional MBS models in which Postulate B is true, even although $\neg$ Postulate A and NOFINFB are true. It is an open question whether for such cases to arise one needs a set $S_{M}$ which is not bounded on the temporal coordinate.

\footnotetext{
${ }^{11} \mathrm{~A}$ reader might want to directly see an odd set $X$ of which Postulate B is true. To this end, from each $e_{n} \in S_{M}$ go up a bit (say, by $1 / 2$ on the temporal axis) along the backward light cone of $x^{*}$ and with the resulting point $x_{n} \in \Re^{4}$ associate a label $g$ such that $\mathrm{g}(\mathrm{n})=0$. The totality of $\left[x_{n} g\right](n \in \mathbb{N})$ is the sought-for $X$.
} 


\section{Conclusion and open problems}

In this paper we have introduced the notion of a Minkowskian Branching Structure, based on Müller (2002). In the second part of the paper we have shown some results concerning finitary and infinitary funny business. There are exactly two ways of generating INFFB which does not involve FINFB: via Postulate A or via Postulate B. On the other hand, the falsity of both postulates in a model with space-time points and NOFINFB entails that there is NOINFFB in this model. In the third part of the paper we explored under what conditions there could be INFFB in MBS. We first observed that if in an MBS model choice points are 'nicely' distributed in $\Re^{4}$, so that condition 26 is true, and NOFINFB is true, then there is no case of INFFB in that model. Also, we have seen that in MBS' a set $S$ responsible for funny business must be quite particularly located, namely if there is funny business produced by a pairwise SLR subset $S$ of a history, then there is some $x \in \Re^{4}$ such that either (1) there is a set $S^{\prime} \subseteq S$, which gives rise to funny business and whose reduced set $S_{M}^{\prime}$ is located on the backward light cone of $x$, or (2) any outer lining of the backward light cone of $x$ comprises the reduced set of a set $S(\delta) \subseteq S$ which generates funny business. Our next finding is that Postulate A can be true in MBS' (and generate INFFB) only provided that there is a convergent sequence in reduced set $S_{M}$ of $S$ which gives rise to INFFB. Finally, we have shown an MBS model in which Postulate B is true, yet Postulate A and FINFB do not hold; the set $X$ of which Postulate B speaks is not bounded on the time coordinate. We conjecture that for Postulate B to be true in an MBS, 'its' set $X$ must have that feature. The moral of our findings is that, spatio-temporally speaking, INFFB without FINFB is possible; however, since this phenomenon requires either convergent (in $\Re^{4}$ ) sequences of choice points, or choice points escaping to infinity on the time coordinate, INFFB without FINFB does not seem to be physically possible. 


\section{Appendix}

\subsection{Splitting points and choice points}

Since it purports to establish that "For histories $h_{\sigma}, h_{\eta} \subset B$ the set $C_{\sigma, \eta}$ is the set of choice points", Lemma 4 in Müller seems to require reformulation. A splitting point, as a member of $\mathbb{R}^{4}$, is not a member of $B$, and thus is not a choice point.

An obvious move would be to observe that every splitting point $x$ for scenarios $\sigma$ and $\eta$ in a sense "generates" a choice point for histories $h_{\sigma}$ and $h_{\eta}$. That is, if $x \in C_{\sigma \eta}$ then $\left[x_{\sigma}\right]$ is maximal in $h_{\sigma} \cap h_{\eta}$.

What might not be as evident is that, since we have dropped the requirement of finitude of every $C_{\sigma \eta}$, the converse is not true: in some cases there are choice points which are not "generated" in the above way by any splitting points. We will now try to persuade the reader that this is indeed the case. The idea is to use sequences of generated splitting points convergent to the same point. The argument is simple in $\mathbb{R}^{2}$ as we need only two sequences, but gets more complicated as the number of dimensions increases. (For convenience, in the below argument we use symbols " $>_{S}$ " and " $>_{M}$ " defined in the natural way basing on respectively " $\leqslant S$ " and " $\leqslant_{M}$ ".)

Definition 34 1. $S C_{\sigma \eta}:=\left\{\left[c_{\sigma}\right] \mid c \in C_{\sigma \eta}\right\}$

$$
\begin{aligned}
2 . \boldsymbol{C}_{\sigma \eta}:=\left\{\left[x_{\gamma}\right]:\right. & \text { (1) }\left[x_{\gamma}\right] \in h_{\sigma} \cap h_{\eta} \text { and } \\
& \text { (2) } \forall z \in \mathbb{R}^{4} \forall \alpha \in \Sigma\left(\left[z_{\alpha}\right]>_{S}\left[x_{\gamma}\right] \Rightarrow\left[z_{\alpha}\right] \notin h_{\sigma} \cap h_{\eta}\right)
\end{aligned}
$$

" $S C_{\sigma \eta}$ " is to be read as "The set of generated choice points for histories $h_{\sigma}$ and $h_{\eta} "$.

" $\mathbf{C}_{\sigma \eta}$ " is to be read as "The set of choice points for histories $h_{\sigma}$ and $h_{\eta}$ ".

It is of course irrelevant whether we choose $\sigma$ or $\eta$ in square brackets in the definition of the set of generated choice points, since if $c \in C_{\sigma \eta}$ then $c_{\sigma} \equiv_{S} c_{\eta}$ and thus $\left[c_{\sigma}\right]=\left[c_{\eta}\right]$.

Theorem 35 For some $C_{\sigma \eta}, S C_{\sigma \eta} \varsubsetneqq \boldsymbol{C}_{\sigma \eta}$.

Proof sketch. Again, by fixing two spatial dimensions we will restrict ourselves to $\mathbb{R}^{2}$. Let $x=(0,0)$. Let $C_{1}=\{(0,1 / n) \mid n \in \mathbb{N} \backslash\{0\}\}$ and $C_{2}=$ $\{(0,-1 / n) \mid n \in \mathbb{N} \backslash\{0\}\}$. Let $C_{\sigma \eta}=C_{1} \cup C_{2}$. As $x \notin C_{\sigma \eta}$, it is evident that $\left[x_{\sigma}\right] \notin S C_{\sigma \eta}$. We will show that $\left[x_{\sigma}\right] \in \mathbf{C}_{\sigma \eta}$, thus proving the theorem.

We have to show that $\left[x_{\sigma}\right]$ meets conditions (1) and (2) from the above definition. As for (1), $\forall c \in C_{\sigma \eta} \mathrm{x} \operatorname{SLR} \mathrm{c}$, so $x \in R_{\sigma \eta}$. It follows that $x_{\sigma} \equiv_{S} x_{\eta}$ and finally (as it is obvious that $\left[x_{\sigma}\right] \in h_{\sigma}$ ) that $\left[x_{\sigma}\right] \in h_{\sigma} \cap h_{\eta}$. 
Now for (2). Consider $\left[z_{\alpha}\right]$ such that (a) $\left[z_{\alpha}\right]>_{S}\left[x_{\sigma}\right]$. By definition of $>_{S}, z>_{M} x$ and $x_{\alpha}=x_{\sigma}$. Let $z=\left(z_{0}, z_{1}\right)$ (the first coordinate is temporal). We distinguish two cases: either the spatial coordinate $z_{1}$ is equal to 0 or it's something else.

If $z=\left(z_{0}, 0\right)$, take $k \in \mathbb{R}, k<z_{0}$ such that $(0, k) \in C_{\sigma \eta}$ (such $k$ exists since $C_{1}$ converges to $\left.(0,0)\right)$. $\left(^{*}\right)$ Since $D_{M}^{2}(z,(0, k))=k-z_{1}<0$, it follows that $x>_{M}(0, k) \in C_{\sigma \eta}$.

On the other hand, if $z_{1} \neq 0$, consider $\mathrm{v}$ defined as follows:

$$
v:= \begin{cases}1 & \text { if } z_{1} \geq 1 \\ z_{1} & \text { if } z_{1} \in(0,1) \cup(-1,0) \\ -1 & \text { if } z_{1} \leqslant-1\end{cases}
$$

We choose $(0, k) \in C_{\sigma \eta}$ such that $0<k \leqslant v$ (if $v$ is positive) or $v \leqslant k<0$ (if $v$ is negative). It is always possible to find such a point $\operatorname{since}$ both $C_{1}$ and $C_{2}$ converge to $(0,0)$. We have to prove that (b) $z>_{M}(0, k)$.

From (a) we know that (c) $z>_{M}(0,0)$. To arrive at (b) it suffices to show that (d) $z>_{M}(0, v)$. From (c) it follows that (e) $z_{0} \geq z_{1}$. We have two cases to consider. First, if (f) $z_{1} \geq 1$ or $z_{1} \leqslant-1, D_{M}^{2}(z,(0, v))=-z_{0}^{2}+\left(z_{1}-1\right)^{2}=$ $-z_{0}^{2}+z_{1}^{2}+1-2 z_{1}$, which (by (f) and (e)) is below 0 , which fact is equivalent to (d). Second, if $z_{1} \in(0,1) \cup(-1,0), D_{M}^{2}(z,(0, v))=-z_{0}^{2}+\left(z_{1}-z_{1}\right)^{2}=-z_{0}^{2}$ which is of course negative, so again we arrive at (d).

From $(\mathrm{c})$ and $(\mathrm{d})$ and from the requirement on choosing $(0, k)$ we get the needed result $(\mathrm{b})$.

Since $z>_{M}(0, k) \in C_{\sigma \eta}$, it is true that $z \notin R_{\sigma \eta}$ and thus $\left[z_{\alpha}\right] \notin h_{\sigma} \cap h_{\eta}$. We have thus proved that $\left[x_{\sigma}\right]$ fulfills condition $(2)$.

Unfortunately already in $\mathbb{R}^{3}$ the construction fails at point $\left(^{*}\right)$. To overcome the problem we would have to use four sequences of splitting points convergent to $(0,0,0)$ (intuitively situated at the arms of the coordinate system). To deal with the situation in $\mathbb{R}^{4}$ we would have to similarly introduce six sequences convergent to $(0,0,0,0)$. We don't dwell into the details here as the point being made doesn't seem to be significant enough in proportion to the arduous complexity of the argument.

Conjecture 36 For any scenarios $\sigma, \eta \in \Sigma$, the set $\boldsymbol{C}_{\sigma \eta}$ contains exclusively points which belong to $S C_{\sigma \eta}$ or points $\left[x_{\alpha}\right]$ such that $x$ is a limit of a sequence of points belonging to $C_{\sigma \eta}$.

\subsection{When the topological postulate is false}

We will now show a situation in which lemma 8 does not hold. The construction resembles the $M_{1}$ structure from Müller et al. (2006). By fixing two 
spatial dimensions we will restrict ourselves to $\mathbb{R}^{2}$, the first coordinate representing time.

As usual, $\Sigma$ is the set of all scenarios of a world $B$. Let $C$ be the set of all splitting points:

$$
C:=\bigcup_{\sigma, \eta \in \Sigma} C_{\sigma \eta}
$$

We put

$$
C:=\{\langle 0, n\rangle \mid n \in \mathbb{N} \cup\{0\}\}
$$

The idea is that all splitting points are binary: any scenario passing through a given splitting point can go either "left" or "right". Since there are as many splitting points as natural numbers, we can identify $\Sigma$ with a set of 01sequences. Another requirement on $\Sigma$ is that it contains only the sequences with finitely many 0 s. Let $G$ be a subset of $\Sigma$ containing only the sequence without any 0s and all sequences that have all their 0s in the beginning. The elements of $G$ will be labeled as below:

$$
\begin{gathered}
\sigma_{0}=1111 \ldots . \\
\sigma_{1}=01111 \ldots \\
\sigma_{2}=00111 \ldots \\
\sigma_{3}=00011 \ldots
\end{gathered}
$$

Let us next consider a sequence $Z_{i}^{M}$ of points in $\mathbb{R}^{2}$ such that for all $i \in$ $\mathbb{N} z_{i}=\langle i-1 / 2,0\rangle$. This way, a given $z_{i} \in Z_{i}^{M}$ is in the Minkowskian sense above all splitting points $\langle 0, n\rangle \mid n<i$ and above no other splitting points.

Consider now a sequence $Z_{i}$ in $B, Z_{i}=\left\{\left[z_{i} \sigma_{i}\right] \mid i \in \mathbb{N}\right\}$ 12. We will now show that $Z_{i}$ is a chain. Take any $\left[z_{m} \sigma_{m}\right],\left[z_{n} \sigma_{n}\right] \in Z_{i}$ such that $m \neq n$. Either $m<n$ or $n<m$; suppose $m<n$ (the other case is analogous). Since $m<n, z_{m} \leqslant_{M} z_{n} . z_{m} \in R_{\sigma_{m} \sigma_{n}}$ since it is not above any splitting points between $\sigma_{m}$ and $\sigma_{n}$. Therefore $z_{m} \sigma_{m} \equiv_{S} z_{m} \sigma_{n}$, so $\left[z_{m} \sigma_{m}\right] \leqslant_{S}\left[z_{n} \sigma_{n}\right]$. We have shown that any two elements of $Z_{i}$ are comparable by $\leqslant_{S}$. Therefore, $Z_{i}$ is a chain in $B$, thus being an upward-directed subset of $B$.

The set of all upward-directed subsets of $B$ meets the requirements of Kuratowski-Zorn Lemma, since a set-theoretical sum of any chain subset of it is also an upward-directed subset of $B$ and is an upper bound of the chain with respect to inclusion. Therefore, there exists a maximal upward-directed subset of $B$ (a history $h^{*}$ ) such that $Z_{i} \subseteq h^{*}$. But lemma 8 is false with respect to this history, since for all $\sigma \in \Sigma, h^{*} \neq\left\{\left[x_{\sigma}\right] \mid x \in \mathbb{R}^{2}\right\}$ ! Suppose to the contrary, that for a certain $\sigma \in \Sigma h=\left\{\left[x_{\sigma}\right] \mid x \in \mathbb{R}^{2}\right\}$. As a member

\footnotetext{
${ }^{12}$ Again, we hope it is clear from the context that $z_{i} \in \mathbb{R}^{4}$ and $\sigma_{i} \in \Sigma$. For clarity, we do not want to write the scenario as a subscript.
} 
of $\Sigma, \sigma$ has to contain a "1" at some point $k$ (starting with 0 ). Then both $\left[z_{k+1} \sigma_{k+1}\right] \in h^{*}$ and $\left[z_{k+1} \sigma\right] \in h^{*}$, so $z_{k+1} \in R_{\sigma_{k} \sigma_{k+1}}$. But $C_{\sigma_{k} \sigma_{k+1}} \ni\langle 0, k\rangle \leqslant_{M}$ $z_{k+1}$, so $z_{k+1} \notin R_{\sigma_{k} \sigma_{k+1}}$ and thus we arrive at a contradiction.

We will now show that our topological postulate 6 is not met in this situation. Consider a chain $Z:=Z_{i}^{M} \cup\{\langle-1,0\rangle\}$. Note that $\langle-1,0\rangle=$ inf $f(Z)$. Consider next the chain topology on $\Sigma_{h^{*}}(\langle-1,0\rangle)$ (as defined in the last section) with $Z$ as the original chain. $\left\{\Sigma_{h^{*}}\left(z_{i}\right)\right\}$ is a centred family of closed sets, but its intersection is empty as $\Sigma$ does not contain a scenario corresponding to the sequence comprised of 0 s only. Therefore we arrived at a contradiction with our corollary 7, so the postulate 6 is not met: the chain topology is not compact.

\section{Equivalences}

We will show that our notion of finitary funny business is equivalent to the Belnap (2002) notion of generalized primary slr modal-correlation funny business and our infinitary funny business is 'almost' equivalent to the Müller et al. (2006) notion of combinatorial funny business.

To recall first Belnap's definition, he says that two initial events $A$ and $B$ and their two elementary possibilities $\Pi_{A}\left\langle h_{A}\right\rangle$ and $\Pi_{B}\left\langle h_{B}\right\rangle$, resp., constitute a case of generalized primary slr modal-correlation funny business iff $A S L R B$ and $\Pi_{A}\left\langle h_{A}\right\rangle \cap \Pi_{B}\left\langle h_{B}\right\rangle=\emptyset$, where $A \subset h_{A}$ and $B \subset h_{B}$ (initial event is an upper bounded subset of a history).

Lemma 37 If $A$ and $B$ and their two elementary possibilities $\Pi_{A}\left\langle h_{A}\right\rangle$ and $\Pi_{B}\left\langle h_{B}\right\rangle$, resp., constitute a case of generalized primary slr modal-correlation funny business, then any $\langle S, f\rangle$ such that $A \cup B \subseteq S$ and $f \in \prod_{e \in S} \Pi_{e}$ and $f(e)=\Pi_{e}\left\langle h_{A}\right\rangle$ if $e \in A$ and $f(e)=\Pi_{e}\left\langle h_{B}\right\rangle$ if $e \in B$, is a case of finitary funny business.

The proof is obvious.

Lemma 38 If $\langle S, f\rangle$ is a case of finitary funny business, then there are $A, B \subset S$ and their two elementary possibilities, resp., $\Pi_{A}\left\langle h_{A}\right\rangle, \Pi_{B}\left\langle h_{B}\right\rangle$ which constitute a case of generalized primary slr modal-correlation funny business.

Proof: From the definition of finitary funny business, we have $A$ and $B$ such that $A S L R B$ and $\bigcap\{f(e): e \in A\} \neq \emptyset, \bigcap\{f(e): e \in B\} \neq \emptyset$. For $h_{A}$ take then any history from the first intersection, and for $h_{B}$ any history from the other. Hence $A \subset h_{A}$ and $B \subset h_{B}$. From $\bigcap\{f(e): e \in A \cup B\}=\emptyset$ it follows that $\Pi_{A}\left\langle h_{A}\right\rangle \cap \Pi_{B}\left\langle h_{B}\right\rangle=\emptyset$. 
Turning next to combinatorial funny business, Müller et al. (2006) define it in terms of a set $T$ of elementary transition, where elementary transition is a pair: $\left\langle\right.$ point event $e_{i}$, elementary possibility $H_{i}$ at $\left.e_{i}\right\rangle$. Thus, a given $\langle S, f\rangle$ uniquely specifies a set of of transitions, and a set of transition uniquely determines a pair $\langle S, f\rangle$. To ease the exposition, in the lemmas below we will refer to $\langle S, f\rangle$ as a set of transitions, and we will claim that under certain condition, $\langle S, f\rangle$ is a case of infinitary funny business iff $\langle S, f\rangle$ is a case of combinatorial funny business.

Müller et al. (2006) say that set $T$ of elementary transitions is combinatorially consistent iff for any two transitions $t_{i}, t_{j} \in T$, (1) if $e_{i}=e_{j}$, then $H_{i}=H_{j}$, (2) if $e_{i}<e_{j}$, then $H_{\left(e_{j}\right)} \subseteq H_{i}$, (3) if $e_{j}<e_{i}$, then $H_{\left(e_{i}\right)} \subseteq H_{j}$, and (4) if $e_{i}$ and $e_{j}$ are incomparable, then $e_{i} S L R e_{j}$.

Finally, they say that $T$ is consistent iff $\emptyset \neq H_{T}:=\bigcap\left\{H_{i} \mid H_{i} \in t_{i} \wedge t_{i} \in\right.$ $T\}$ and that $T$ constitutes a case of combinatorial funny business iff $T$ is combinatorially consistent but inconsistent.

We finally turn to our lemmas:

Lemma 39 If $\langle S, f\rangle$ is a case of infinitary funny business, then $\langle S, f\rangle$ constitutes a case of combinatorial funny business.

Proof: We need first to show that $\langle S, f\rangle$ is consistent. Since $f$ is a function, condition (1) of combinatorial consistency holds. Conditions (2) and (3) of combinatorial consistency follow from the condition: $\forall e, e^{\prime} \in S\left(e<e^{\prime} \rightarrow\right.$ $f\left(e^{\prime}\right) \subseteq f(e)$ ) of infinitary funny business. (Suppose $e<e^{\prime}$. Let $f\left(e^{\prime}\right)=H_{j}$ and $f(e)=H_{i}$. Since $f\left(e^{\prime}\right) \subseteq f(e)$, then $H_{j} \subseteq H_{i}$. We need to prove that $H_{\left(e^{\prime}\right)} \subseteq H_{i}$. Suppose to the contrary that (a) there is a history $h^{*} \in H_{\left(e^{\prime}\right)}-H_{i}$. Since $e<e^{\prime}$, there exists a history $h \in H_{e^{\prime}} \cap H_{i}$. From the fact that $e^{\prime} \in h \cap h^{*}$ we get that $h \equiv_{e} h^{*}$, therefore $h^{*} \in H_{i}$ and we arrive at a contradiction with (a).) Condition (4) of combinatorial consistency comes from the condition $\forall A\left(A \subset_{f i n} S \rightarrow \exists h \in\right.$ Hist $\left.: A \subset h\right)$ of infinitary funny business. Finally, since $H_{T}=\bigcap\{f(e): e \in S\}$, by $\bigcap\{f(e): e \in S\}=\emptyset$ we get $H_{T}=\emptyset$.

Lemma 40 If $\langle S, f\rangle$ constitutes a case of combinatorial funny business but $\langle S, f\rangle$ is not a case of finitary funny business, then $\langle S, f\rangle$ is a case of infinitary funny business.

Proof: From $H_{T}=\emptyset$ and $H_{T}=\bigcap\{f(e): e \in S\}$, it must be that $\bigcap\{f(e)$ : $e \in S\}=\emptyset$. Conditions (2) and (3) of consistency entail condition: $\forall e, e^{\prime} \in$ $S\left(e<e^{\prime} \rightarrow f\left(e^{\prime}\right) \subseteq f(e)\right)$ of infinitary funny business. And, if $\operatorname{card}(S)<\omega$, by condition (4) of combinatorial consistency and the assumption that $\langle S, f\rangle$ is not a case of finitary funny business, we get that $\langle S, f\rangle$ is consistent, i.e., not a case of combinatorial funny business. Thus, $\operatorname{card}(S) \geqslant \omega$. 


\section{Location of choice points}

Consider Prior Choice Principle. It says that if (0) $x \in h / h^{\prime}$, then there is $e$ such that (1) $e<x$ and (2) $h^{\prime} \perp_{e} h$. (0) - (2) then entail that $\forall h x \in$ $h \rightarrow h^{\prime} \perp_{e} h$, which can be written as $h^{\prime} \perp_{e} H_{(x)}$. Thus, defining $C_{h^{\prime}}(x):=$ $\left\{e \in W \mid h^{\prime} \perp_{e} H_{(x)}\right\}$, we already know that if $C_{h^{\prime}}(x)$ is non-empty, then by (1) at least one of its elements is below $x$. Where are other elements of $C_{h^{\prime}}(x)$ located? Our claim now is that if $(3) C_{h^{\prime}}(x) \cup\{x\}$ does not give rise to FINFB, then $C_{h^{\prime}}(x)<x$. For reductio, suppose that $\left.h^{\prime} \perp_{e^{\prime}} H_{(x)}\right\}$ and $e^{\prime} S L R x$. By the assumption (3), there is $h^{\prime \prime} \in \Pi_{e^{\prime}}\left\langle h^{\prime}\right\rangle \cap H(x)$, and hence $h^{\prime} \equiv{ }_{e^{\prime}} h^{\prime \prime}$ and $h^{\prime \prime} \in H_{(x)}$, which contradicts $h^{\prime} \perp_{e^{\prime}} H_{(x)}$.

For a future reference, we will generalize slightly these observations and put it as a fact:

Fact 41 If $C(x):=\left\{e \in W \mid \exists h: h \perp_{e} H_{(x)}\right\}$ is non-empty and $C(x) \cup\{x\}$ does not give rise to FINFB, then $C(x)<x$.

It is worth noting that the fact is a special case of one of equivalences of four various notions of modal funny business that Belnap (2002) and Belnap (2003) established. It is a special case of the implication from the so-called some-cause-like-locus-not-in-past funny business to generalized primary shr modal-correlation funny business.

\section{References}

Belnap, N. (1992). Branching space-time. Synthese, 92:385-434. 'Postprint' archived at PhilSci Archive, http://philsci-archive.pitt.edu/archive/00001003.

Belnap, N. (2002). EPR-like "funny business" in the theory of branching space-times. In T. Placek and J. Butterfield (eds.), Nonlocality and Modality, NATO Science Series, pp. 293-315, Dordrecht. Kluwer Academic Publisher.

Belnap, N. (2003). No-common-cause EPR-like funny business in branching space-times. Philosophical Studies, 114:199-221.

Belnap, N. and Szabó, L. (1996). Branching space-time analysis of the GHZ theorem. Foundations of Physics, 26(8):982-1002.

Bohm, D. (1951). Quantum Theory. Prentice-Hall, New York. 
Einstein, A., Podolsky, B., and Rosen, N. (1935). Can quantum-mechanical description of physical reality be considered complete? Physical Review, 47:777-780.

Greenberger, D., Horne, M., and Zeilinger, A. (1989). Going beyond Bell's theorem. In M. Kafatos (ed.), Bell's Theorem, Quantum Theory, and Conceptions of Universe, pp. 69-74. Kluwer Academic Publishers, Dordrecht.

Mermin, N. (1990). What's wrong with these elements of reality? Physics Today, 43:9-11.

Müller, T. (2002). Branching space-time, modal logic and the counterfactual conditional. In T. Placek and J. Butterfield (eds.), Nonlocality and Modality, NATO Science Series, pp. 273-291, Dordrecht. Kluwer Academic Publisher.

Müller, T. (2005). Probability theory and causation: a Branching SpaceTimes analysis. British Journal for the Philosophy of Science, 56(3):487520.

Müller, T., Belnap, N., and Kishida, K. (2006). Funny business in branching space-times: Infinite modal correlations. archived at: http://philsci-archive.pitt.edu/archive/00002803/.

Placek, T. (2000). Stochastic outcomes in branching space-time. An analysis of the Bell theorems. British Journal for the Philosophy of Science, $51(3): 445-475$. 